TRANSACTIONS OF THE

AMERICAN MATHEMATICAL SOCIETY

Volume 361, Number 12, December 2009, Pages 6407-6428

S 0002-9947(09)04913-7

Article electronically published on June 5, 2009

\title{
ON CLASSES OF BANACH SPACES ADMITTING "SMALL" UNIVERSAL SPACES
}

\author{
PANDELIS DODOS
}

\begin{abstract}
We characterize those classes $\mathcal{C}$ of separable Banach spaces admitting a separable universal space $Y$ (that is, a space $Y$ containing, up to isomorphism, all members of $\mathcal{C}$ ) which is not universal for all separable Banach spaces. The characterization is a byproduct of the fact, proved in the paper, that the class NU of non-universal separable Banach spaces is strongly bounded. This settles in the affirmative the main conjecture from Argyros and Dodos (2007). Our approach is based, among others, on a construction of $\mathcal{L}_{\infty}$-spaces, due to J. Bourgain and G. Pisier. As a consequence we show that there exists a family $\left\{Y_{\xi}: \xi<\omega_{1}\right\}$ of separable, non-universal, $\mathcal{L}_{\infty}$-spaces which uniformly exhausts all separable Banach spaces. A number of other natural classes of separable Banach spaces are shown to be strongly bounded as well.
\end{abstract}

\section{INTRODUCTION}

(A) Universality problems have been posed in Banach Space Theory from its early beginnings. A typical one can be stated as follows.

(P1) Let $\mathcal{C}$ be a class of separable Banach spaces. When can we find a space $Y$ that belongs in the class $\mathcal{C}$ and contains an isomorphic copy of every member of $\mathcal{C}$ ?

A space $Y$ containing an isomorphic copy of every member of $\mathcal{C}$ is called a universal space of the class. As it turns out, the requirement that the universal space of $\mathcal{C}$ lies also in $\mathcal{C}$ is quite restrictive. Consider, for instance, the class UC of all separable uniformly convex Banach spaces. It is easy to see that if $Y$ is any separable space universal for the class UC, then $Y$ cannot be uniformly convex. However, as was shown by E. Odell and Th. Schlumprecht $\mathrm{OS}$, there exists a separable reflexive space $R$ containing an isomorphic copy of every separable uniformly convex space (see also $[\mathrm{DF}]$ ). Keeping this example in mind, we see that in order to have nontrivial answers to problem (P1), we should relax the demand that the universal space $Y$ of the class $\mathcal{C}$ is in addition a member of $\mathcal{C}$. Instead, we should look for "small" universal spaces. The most natural requirement, at this level of generality, is to ensure that the universal space of $\mathcal{C}$ is not universal for all separable Banach spaces. So, one is led to face the following problem.

Received by the editors October 18, 2007.

2000 Mathematics Subject Classification. Primary 03E15, 46B03, 46B07, 46B15.

Key words and phrases. Non-universal spaces, strongly bounded classes, Schauder bases, $\mathcal{L}_{\infty^{-}}$ spaces.

(C)2009 American Mathematical Society Reverts to public domain 28 years from publication 6407 
(P2) Let $\mathcal{C}$ be a class of separable Banach spaces. When can we find a separable space $Y$ which is universal for the class $\mathcal{C}$ but not universal for all separable Banach spaces?

(B) A first necessary condition a class $\mathcal{C}$ must satisfy in order to admit a "small" universal space ("small" in the sense of (P2) above) can be traced in the work of J. Bourgain Bou1] in the early 1980s. To describe it, let us consider the space $C\left(2^{\mathbb{N}}\right)$, where $2^{\mathbb{N}}$ stands for the Cantor set, and let us fix a normalized Schauder basis $\left(e_{n}\right)$ of $C\left(2^{\mathbb{N}}\right)$. Bourgain associated to every separable Banach space $X$ an ordinal, which we shall denote by $\phi_{\mathrm{NU}}(X)$, measuring how well the initial segments of the basis $\left(e_{n}\right)$ are placed inside the space $X$ (the precise definition of $\phi_{\mathrm{NU}}(X)$ is given in $\S 2$ ). This ordinal index $\phi_{\mathrm{NU}}$ satisfies two basic properties. The first property is that it characterizes separable non-universa 11 spaces, in the sense that a separable Banach space $X$ is non-universal if and only if $\phi_{\mathrm{NU}}(X)<\omega_{1}$. The second one is that it is monotone with respect to subspaces. That is, if $X$ is isomorphic to a subspace of $Y$, then $\phi_{\mathrm{NU}}(X) \leq \phi_{\mathrm{NU}}(Y)$. Combining these two properties, we arrive at the following necessary condition for an affirmative answer to problem (P2).

(C1) Let $\mathcal{C}$ be a class of separable Banach spaces admitting a universal space $Y$ which is not universal for all separable Banach spaces. Then the index $\phi_{\mathrm{NU}}$ is uniformly bounded on $\mathcal{C}$, as $\sup \left\{\phi_{\mathrm{NU}}(X): X \in \mathcal{C}\right\} \leq \phi_{\mathrm{NU}}(Y)<\omega_{1}$.

(C) A second necessary condition for an affirmative answer to problem (P2) can be found in the work of B. Bossard Bos1] in the 1990s. Before we state it, let us briefly recall the general framework of Bossard's work. Let $F\left(C\left(2^{\mathbb{N}}\right)\right)$ be the set of all closed subsets of $C\left(2^{\mathbb{N}}\right)$ and consider the set

$$
\mathrm{SB}=\left\{X \in F\left(C\left(2^{\mathbb{N}}\right)\right): X \text { is a linear subspace }\right\} .
$$

Endowing the set SB with the relative Effors-Borel structure, the space SB becomes a standard Borel space. As $C\left(2^{\mathbb{N}}\right)$ is isometrically universal for all separable Banach spaces, we may identify any class of separable Banach spaces with a subset of SB. Under this point of view, we denote by NU the subset of SB consisting of all $X \in \mathrm{SB}$ which are non-universal.

It is relatively easy to see that for every $Y \in \mathrm{SB}$ the set $S(Y)=\{X \in \mathrm{SB}$ : $X$ is isomorphic to a subspace of $Y\}$ is analytic. This observation leads to a second necessary condition for an affirmative answer to problem (P2).

(C2) Let $\mathcal{C}$ be a class of separable Banach spaces admitting a universal space $Y$ which is not universal for all separable Banach spaces. Then there exists an analytic subset $A$ of $\mathrm{NU}$ with $\mathcal{C} \subseteq A$; simply take $A=S(Y)$.

(D) One of the main goals of the present paper is to show that conditions (C1) and (C2) stated above are not only necessary for an affirmative answer to problem (P2) but they are also sufficient. Precisely, the following is proved.

Theorem 1. Let $\mathcal{C}$ be a subset of $\mathrm{SB}$. Then the following are equivalent.

(i) The class $\mathcal{C}$ admits a separable universal space $Y$ which is not universal for all separable Banach spaces.

(ii) We have $\sup \left\{\phi_{\mathrm{NU}}(X): X \in \mathcal{C}\right\}<\omega_{1}$.

(iii) There exists an analytic subset $A$ of $\mathrm{NU}$ with $\mathcal{C} \subseteq A$.

\footnotetext{
${ }^{1} \mathrm{~A}$ Banach space is non-universal if it is not universal for all separable Banach spaces.
} 
Theorem 1 is actually a consequence of a structural property of the class NU of all non-universal separable Banach spaces. To state it we recall the following notion, introduced in $\mathrm{AD}$.

Definition 2. A class $\mathcal{C}$ is said to be strongly bounded if for every analytic subset $A$ of $\mathcal{C}$ there exists $Y \in \mathcal{C}$ that contains an isomorphic copy of every $X \in A$.

The main result of the paper is the following.

Theorem 3. The class NU is strongly bounded.

The problem whether the class NU is strongly bounded had been asked by Alekos Kechris in the 1980s.

(E) Another consequence of Theorem 3 is related to the following notions.

Definition $4([\mathrm{AD}])$. Let $\mathcal{C}$ be an isomorphic invariant class of separable Banach spaces such that every $X \in \mathcal{C}$ is not universal.

(1) We say that the class $\mathcal{C}$ is Bourgain generic if every separable Banach space $Y$ which is universal for the class $\mathcal{C}$ must be universal for all separable Banach spaces.

(2) We say that the class $\mathcal{C}$ is Bossard generic if every analytic set $A$ that contains all members of $\mathcal{C}$ up to isomorphism must contain a $Y \in A$ which is universal for all separable Banach spaces.

It is easy to see that if a class $\mathcal{C}$ of separable Banach spaces is Bossard generic, then it is also Bourgain generic. In $\mathrm{AD}$ it was conjectured that the above notions coincide. Theorem 3 settles this in the affirmative.

Corollary 5. Bourgain genericity coincides with Bossard genericity.

(F) The proof of Theorem 3 relies, in part, on some of the results proved in [AD], in particular on the fact that the class of all non-universal spaces with a Schauder basis is strongly bounded (this material is recalled in §5). The new component is the use of a construction of $\mathcal{L}_{\infty}$-spaces, due to Jean Bourgain and Gilles Pisier BP. The Bourgain-Pisier construction was the outcome of the combination of two major achievements of Banach Space Theory during the 1980s. The first one is the Bourgain-Delbaen space [BD], the first example of an $\mathcal{L}_{\infty}$-space not containing an isomorphic copy of $c_{0}$. The second one is Pisier's scheme [Pi] for producing counterexamples to a conjecture of Grothendieck. A striking similarity between the two methods, which is reflected in the Bourgain-Pisier construction, is that they both produce infinite-dimensional spaces essentially by developing techniques for extending finite-dimensional ones.

The following consequence of Theorem 3 provides a more accurate insight of the reasoning behind the proof of Theorem 3.

Corollary 6. For every $\lambda>1$ there exists a family $\left\{Y_{\xi}^{\lambda}: \xi<\omega_{1}\right\}$ of separable Banach spaces with the following properties.

(i) For every $\xi<\omega_{1}$ the space $Y_{\xi}^{\lambda}$ is non-universal and $\mathcal{L}_{\infty, \lambda+}$.

(ii) If $\xi<\zeta<\omega_{1}$, then $Y_{\xi}^{\lambda}$ is contained in $Y_{\zeta}^{\lambda}$.

(iii) If $X$ is a separable space with $\phi_{\mathrm{NU}}(X) \leq \xi$, then $X$ is contained in $Y_{\xi}^{\lambda}$.

Corollary [6] shows that the class of $\mathcal{L}_{\infty}$-spaces is "generic". This result was somehow surprising to the author, as he thought that a typical separable Banach 
space "looks like" Tsirelson's space Ts. Recent work, however, of E. Odell, Th. Schlumprecht and A. Zsák OSZ shows that a typical separable reflexive space is indeed a Tsirelson space.

(G) Although in AD] a number of natural classes of separable Banach spaces were shown to be strongly bounded, until recently, the only classes which were known to be strongly bounded, without having to impose on them any restriction on the existence of a basis, were the class REFL of separable reflexive spaces and the class SD of spaces with separable dual (see DF].

The final result we would like to mention in the introduction is that there exist continuum many such strongly bounded classes (besides, of course, the classes REFL, SD and NU). Before we give the precise statement let us recall that an infinite-dimensional Banach space $X$ is said to be minimal if $X$ embeds into every infinite-dimensional subspace of it (e.g. the classical sequence spaces $c_{0}$ and $\ell_{p}$ are minimal). We show the following.

Theorem 7. Let $X$ be a minimal Banach space not containing $\ell_{1}$. Then the class

$$
\mathrm{NC}_{X}=\{Y \in \mathrm{SB}: Y \text { does not contain an isomorphic copy of } X\}
$$

is strongly bounded.

\section{BACKGROUND MATERIAL}

Our general notation and terminology is standard as can be found, for instance, in $[\mathrm{LT}]$ and $[\mathrm{Ke}$. By $\mathbb{N}=\{0,1,2, \ldots\}$ we shall denote the natural numbers. By $[\mathbb{N}]$ we denote the set of all infinite subsets of $\mathbb{N}$, which is clearly a $G_{\delta}$, hence Polish, subspace of $2^{\mathbb{N}}$.

2.1. Trees and dyadic subtrees. Let $\Lambda$ be a non-empty set. By $\Lambda^{<\mathbb{N}}$ we shall denote the set of all finite sequences in $\Lambda$. The empty sequence is denoted by $\varnothing$ and is included in $\Lambda^{<\mathbb{N}}$. We view $\Lambda^{<\mathbb{N}}$ as a tree equipped with the (strict) partial order $\sqsubset$ of end-extension. For every $s, t \in \Lambda^{<\mathbb{N}}$ by $s^{\frown} t$ we shall denote their concatenation; by $|t|$ we shall denote the length of $t$, i.e. the cardinality of the set $\left\{s \in \Lambda^{<\mathbb{N}}: s \sqsubset t\right\}$. By $\Lambda^{\mathbb{N}}$ we denote the set of all infinite sequences in $\Lambda$. Equipping $\Lambda$ with the discrete topology and $\Lambda^{\mathbb{N}}$ with the product topology, we see that $\Lambda^{\mathbb{N}}$ is a completely metrizable space which is additionally separable if $\Lambda$ is countable. For every $n, k \in \mathbb{N}$ with $n \leq k$ and every $t \in \Lambda^{<\mathbb{N}}$ with $|t|=k$ we set $t \mid n=(t(0), \ldots, t(n-1))$ if $n \geq 1$, while $t \mid 0=\varnothing$. Similarly, for every $\sigma \in \Lambda^{\mathbb{N}}$ and every $n \in \mathbb{N}$ we set $\sigma \mid n=(\sigma(0), \ldots, \sigma(n-1))$ if $n \geq 1$, while $\sigma \mid 0=\varnothing$.

A tree $T$ on $\Lambda$ is a downwards closed subset of $\Lambda^{<\mathbb{N}}$. By $\operatorname{Tr}(\Lambda)$ we shall denote the set of all trees on $\Lambda$. Hence

$$
T \in \operatorname{Tr}(\Lambda) \Leftrightarrow \forall s, t \in \Lambda^{<\mathbb{N}}(s \sqsubseteq t \text { and } t \in T \Rightarrow s \in T) .
$$

Notice that if $\Lambda$ is countable, then $\operatorname{Tr}(\Lambda)$ is a closed subspace of the compact metrizable space $2^{\Lambda^{<N}}$. The body $[T]$ of a tree $T$ on $\Lambda$ is defined to be the set $\left\{\sigma \in \Lambda^{\mathbb{N}}: \sigma \mid n \in T \forall n \in \mathbb{N}\right\}$. A tree $T$ is said to be pruned if for every $t \in T$ there exists $s \in T$ with $t \sqsubset s$. It is said to be well-founded if $[T]=\varnothing$. The set of all well-founded trees on $\Lambda$ is denoted by $\operatorname{WF}(\Lambda)$. For every $T \in \operatorname{WF}(\Lambda)$ we let $T^{\prime}=\{s \in T: \exists t \in T$ with $s \sqsubset t\} \in \mathrm{WF}(\Lambda)$. By transfinite recursion, we define the iterated derivatives $T^{(\xi)}\left(\xi<\kappa^{+}\right)$of $T$, where $\kappa$ stands for the cardinality of $\Lambda$. The order $o(T)$ of $T$ is defined to be the least ordinal $\xi$ such that $T^{(\xi)}=\varnothing$. 
Let $2^{<\mathbb{N}}$ be the Cantor tree, i.e. the tree consisting of all finite sequences of 0 's and 1's. For every $s, t \in 2^{<\mathbb{N}}$ we let $s \wedge t$ be the $\sqsubset$-maximal node $w$ of $2^{<\mathbb{N}}$ with $w \sqsubseteq s$ and $w \sqsubseteq t$. If $s, t \in 2^{<\mathbb{N}}$ are incomparable with respect to $\sqsubseteq$, then we write $s \prec t$ provided that $(s \wedge t)^{\frown} 0 \sqsubseteq s$ and $(s \wedge t)^{\frown} 1 \sqsubseteq t$. We say that a subset $D$ of $2^{<\mathbb{N}}$ is a dyadic subtree of $2^{<\mathbb{N}}$ if $D$ can be written in the form $D=\left(s_{t}\right)_{t \in 2<\mathbb{N}}$ so that for every $t_{1}, t_{2} \in 2^{<\mathbb{N}}$ we have $t_{1} \sqsubset t_{2}$ (respectively $t_{1} \prec t_{2}$ ) if and only if $s_{t_{1}} \sqsubset s_{t_{2}}$ (respectively $s_{t_{1}} \prec s_{t_{2}}$ ). It is easy to see that such a representation of $D$ as $\left(s_{t}\right)_{t \in 2<\mathbb{N}}$ is unique. In the sequel, when we write $D=\left(s_{t}\right)_{t \in 2<\mathbb{N}}$, where $D$ is a dyadic subtree, we will assume that this is the canonical representation of $D$ described above.

2.2. Operators fixing copies of $C\left(2^{\ltimes}\right)$. Let $X, Y$ and $Z$ be Banach spaces and $T: X \rightarrow Y$ be a bounded linear operator. We say that the operator $T$ fixes a copy of $Z$ if there exists a subspace $E$ of $X$ which is isomorphic to $Z$ and such that $\left.T\right|_{E}$ is an isomorphic embedding. In [Ro1, H. P. Rosenthal has shown that if $X$ is a Banach space and $T: C([0,1]) \rightarrow X$ is an operator such that $T^{*}$ has non-separable range, then $T$ fixes a copy of $C([0,1])$. This result combined with a classical discovery of A. A. Milutin Mi] yields the following.

Theorem 8 ([Ro1]). Let $K$ be an uncountable compact metrizable space, $X$ a Banach space and $T: C(K) \rightarrow X$ a bounded linear operator. If $T$ fixes a copy of $\ell_{1}$, then $T$ also fixes a copy of $C(K)$.

We refer the reader to $\mathrm{Ga}$, Ro3 and the references therein for stronger versions of Theorem 8 .

2.3. $\mathcal{L}_{\infty}$-spaces. We recall that if $X$ and $Y$ are two isomorphic Banach spaces (not necessarily infinite-dimensional), then their Banach-Mazur distance is defined by

$$
d(X, Y)=\inf \left\{\|T\| \cdot\left\|T^{-1}\right\|: T: X \rightarrow Y \text { is an isomorphism }\right\} .
$$

Now let $\lambda \geq 1$. An infinite-dimensional Banach space $X$ is said to be an $\mathcal{L}_{\infty, \lambda \text {-space }}$ if for every finite-dimensional subspace $F$ of $X$ there exists a finite-dimensional subspace $G$ of $X$ with $F \subseteq G$ and such that $d\left(G, \ell_{\infty}^{n}\right) \leq \lambda$ where $n=\operatorname{dim} G$. The space $X$ is said to be an $\mathcal{L}_{\infty, \lambda+}$-space if $X$ is an $\mathcal{L}_{\infty, \theta}$-space for any $\theta>\lambda$. Finally, the space $X$ is said to be an $\mathcal{L}_{\infty}$-space if $X$ is an $\mathcal{L}_{\infty, \lambda}$-space for some $\lambda \geq 1$. The class of $\mathcal{L}_{\infty}$-spaces was introduced by J. Lindenstrauss and A. Pełczyński [LP1].

It follows readily by the above definition that if $X$ is a separable $\mathcal{L}_{\infty, \lambda \text {-space, }}$ then there exists an increasing (with respect to inclusion) sequence $\left(G_{n}\right)$ of finitedimensional subspaces of $X$ with $\bigcup_{n} G_{n}$ dense in $X$ and such that $d\left(G_{n}, \ell_{\infty}^{m_{n}}\right) \leq \lambda$ where $m_{n}=\operatorname{dim} G_{n}$ for every $n \in \mathbb{N}$. It is relatively easy to see that this property actually characterizes separable $\mathcal{L}_{\infty}$-spaces. In particular, if $X$ is a separable Banach space and there exists an increasing sequence $\left(F_{n}\right)$ of finite-dimensional subspaces of $X$ with $\bigcup_{n} F_{n}$ dense in $X$ and such that $d\left(F_{n}, \ell_{\infty}^{m_{n}}\right) \leq \lambda$ where $m_{n}=\operatorname{dim} F_{n}$, then $X$ is an $\mathcal{L}_{\infty, \lambda+}$-space.

The book of J. Bourgain Bou2 contains a presentation of the theory of $\mathcal{L}_{\infty^{-}}$ spaces and a discussion of many remarkable examples. Among the structural properties of $\mathcal{L}_{\infty}$-spaces, the following one, due to W. B. Johnson, H. P. Rosenthal and M. Zippin, will be of particular importance for us.

Theorem 9 ([JRZ]). Every separable $\mathcal{L}_{\infty}$-space has a Schauder basis. 
We refer the reader to Ro3 for a discussion on further properties of $\mathcal{L}_{\infty}$-spaces, as well as for a presentation of refinements of Theorem 9 ,

2.4. Descriptive set-theoretical preliminaries. A standard Borel space is a measurable space $(X, S)$ for which there exists a Polish topology $\tau$ on $X$ such that the Borel $\sigma$-algebra of $(X, \tau)$ coincides with $S$. A classical result in the theory of Borel sets in Polish spaces asserts that if $(X, S)$ is a standard Borel space and $B \in S$, then $B$ equipped with the relative $\sigma$-algebra is also a standard Borel space (see [Ke, Corollary 13.4]).

A basic example of a standard Borel space is the Effros-Borel structure on the set of closed subsets of a Polish space. Specifically, let $X$ be a Polish space and let us denote by $F(X)$ the set of all closed subsets of $X$. We endow $F(X)$ with the $\sigma$-algebra $\Sigma$ generated by the sets

$$
\{F \in F(X): F \cap U \neq \varnothing\},
$$

where $U$ ranges over all open subsets of $X$. It is well known (see $[\mathrm{Ke}$, Theorem 12.6]) that the measurable space $(F(X), \Sigma)$ is standard.

A subset $A$ of a standard Borel space $(X, S)$ is said to be analytic if there exists a Borel map $f: \mathbb{N}^{\mathbb{N}} \rightarrow X$ with $f\left(\mathbb{N}^{\mathbb{N}}\right)=A$. A subset of $(X, S)$ is said to be co-analytic if its complement is analytic. We will adopt the modern, logical, notation to denote these classes. Hence, $\boldsymbol{\Sigma}_{1}^{1}$ stands for the class of analytic sets, while $\boldsymbol{\Pi}_{1}^{1}$ stands for the class of co-analytic ones.

Let us also recall the notion of a $\Pi_{1}^{1}$-rank, introduced by Y. N. Moschovakis. Let $X$ be a standard Borel space and $B$ be a co-analytic subset of $X$. A map $\psi: B \rightarrow \omega_{1}$ is said to be a $\Pi_{1}^{1}$-rank on $B$ if there exist relations $\leq_{\Sigma}, \leq_{\Pi} \subseteq X \times X$ in $\boldsymbol{\Sigma}_{1}^{1}$ and $\boldsymbol{\Pi}_{1}^{1}$ respectively such that for every $y \in B$ we have

$$
x \in B \text { and } \psi(x) \leq \psi(y) \Leftrightarrow x \leq_{\Sigma} y \Leftrightarrow x \leq_{\Pi} y .
$$

In the following lemma we gather all the structural properties of $\boldsymbol{\Pi}_{1}^{1}$-ranks that we need. For a proof, as well as for a thorough presentation of Rank Theory, we refer to [Ke, §34].

Lemma 10. Let $X$ be a standard Borel space, $B$ a co-analytic subset of $X$ and $\psi: B \rightarrow \omega_{1}$ a $\boldsymbol{\Pi}_{1}^{1}$-rank on $B$. Then the following hold.

(i) For every $\xi<\omega_{1}$ the set $B_{\xi}=\{x \in B: \psi(x) \leq \xi\}$ is Borel.

(ii) (Boundedness) If $A \subseteq B$ is analytic, then $\sup \{\psi(x): x \in A\}<\omega_{1}$.

2.5. The standard Borel space of separable Banach spaces. Let $X$ be a separable Banach space (not necessarily infinite-dimensional) and let $(F(X), \Sigma)$ be the Effors-Borel structure on the set of all closed subsets of $X$. Consider the set

$$
\operatorname{Subs}(X)=\{Y \in F(X): Y \text { is a linear subspace }\} \text {. }
$$

It is easy to see that $\operatorname{Subs}(X)$ is a Borel subset of $F(X)$, and so, a standard Borel space on its own. If $X=C\left(2^{\mathbb{N}}\right)$, then we shall denote the space $\operatorname{Subs}\left(C\left(2^{\mathbb{N}}\right)\right)$ by $\mathrm{SB}$ and we shall refer to the space SB as the standard Borel space of all separable Banach spaces. We will need the following consequence of the Kuratowski-RyllNardzewski selection theorem (see [Ke, page 264] for more details).

Proposition 11. Let $X$ be a separable Banach space. Then there exists a sequence $d_{n}: \operatorname{Subs}(X) \rightarrow X(n \in \mathbb{N})$ of Borel maps with $d_{n}(Y) \in Y$ and such that the sequence $\left(d_{n}(Y)\right)$ is norm-dense in $Y$ for every $Y \in \operatorname{Subs}(X)$. 
Let $Z$ be a Banach space with a Schauder basis (throughout the paper, when we say that a Banach space $Z$ has a Schauder basis, then we implicitly assume that $Z$ is infinite-dimensional). We fix a normalized Schauder basis $\left(e_{n}\right)$ of $Z$. Consider the set

$$
\mathrm{NC}_{Z}=\{X \in \mathrm{SB}: X \text { does not contain an isomorphic copy of } Z\} .
$$

Notice that if $Z=C\left(2^{\mathbb{N}}\right)$, then the above-defined class coincides with the class NU of all $X \in \mathrm{SB}$ which are non-universal. Let $\delta>0$ and let $Y$ be an arbitrary separable Banach space. Following J. Bourgain [Bou1, we introduce a tree $\mathbf{T}\left(Y, Z,\left(e_{n}\right), \delta\right)$ on $Y$ defined by the rule

$$
\left(y_{0}, \ldots, y_{k}\right) \in \mathbf{T}\left(Y, Z,\left(e_{n}\right), \delta\right) \Leftrightarrow\left(y_{n}\right)_{n=0}^{k} \text { is } \delta \text {-equivalent to }\left(e_{n}\right)_{n=0}^{k} .
$$

It is easy to see that $Y \in \mathrm{NC}_{Z}$ if and only if for every $\delta>0$ the tree $\mathbf{T}\left(Y, Z,\left(e_{n}\right), \delta\right)$ is well-founded. We set $\phi_{\mathrm{NC}_{Z}}(Y)=\omega_{1}$ if $Y \notin \mathrm{NC}_{Z}$, while if $Y \in \mathrm{NC}_{Z}$ we define

$$
\phi_{\mathrm{NC}_{Z}}(Y)=\sup \left\{o\left(\mathbf{T}\left(Y, Z,\left(e_{n}\right), \delta\right)\right): \delta>0\right\} .
$$

If $Z=C\left(2^{\mathbb{N}}\right)$, then we shall denote by $\phi_{\mathrm{NU}}(Y)$ the above quantity. Although the definition of the ordinal ranking $\phi_{\mathrm{NC}_{Z}}$ depends on the choice of the Schauder basis $\left(e_{n}\right)$ of $Z$, it can be shown that it is actually independent of such a choice in a very strong sense (see $[\mathrm{AD}$, Theorem 10] for more details).

In [Bou1, Bourgain proved that for every Banach space $Z$ with a Schauder basis and every $Y \in \mathrm{SB}$ we have that $Y \in \mathrm{NC}_{Z}$ if and only if $\phi_{\mathrm{NC}_{Z}}(Y)<\omega_{1}$. We need the following refinement of this result.

Theorem 12 (Bos2 $)$. Let $Z$ be a Banach space with a Schauder basis. Then the following hold.

(i) The set $\mathrm{NU}$ is $\boldsymbol{\Pi}_{1}^{1}$ and the map $\phi_{\mathrm{NU}}: \mathrm{NU} \rightarrow \omega_{1}$ is a $\boldsymbol{\Pi}_{1}^{1}$-rank on $\mathrm{NU}$.

(ii) The set $\mathrm{NC}_{Z}$ is $\Pi_{1}^{1}$ and the map $\phi_{\mathrm{NC}_{Z}}: \mathrm{NC}_{Z} \rightarrow \omega_{1}$ is a $\boldsymbol{\Pi}_{1}^{1}$-rank on $\mathrm{NC}_{Z}$.

\section{A result on quotient spaces}

Throughout this section by $X, Y, Z$ and $E$ we shall denote infinite-dimensional Banach spaces. We say that a space $X$ is hereditarily $Y$ if every subspace $Z$ of $X$ contains an isomorphic copy of $Y$. A space $X$ is said to have the Schur property if every weakly convergent sequence in $X$ is automatically norm convergent. It is an immediate consequence of Rosenthal's Dichotomy [Ro2] that a space with the Schur property is hereditarily $\ell_{1}$. The converse is not valid, as shown by J. Bourgain. We will need the following stability result concerning quotient spaces.

Proposition 13. Let $E$ be a minimal Banach space not containing $\ell_{1}$. Also let $X$ be a Banach space and $Y$ be a subspace of $X$. Assume that the quotient $X / Y$ has the Schur property. Then the following hold.

(i) If $Y$ is non-universal, then so is $X$.

(ii) If $Y$ does not contain an isomorphic copy of $E$, then neither does $X$.

Proof. (i) This part is essentially a consequence of a result due to J. Lindenstrauss and A. Pełczyński asserting that the property of not containing an isomorphic copy of $C([0,1])$ is a three-space property [LP2, Theorem 2.1]. For the convenience of the reader, however, we shall give a proof for this special case.

To this end, we need to introduce some pieces of notation. Let $\mathcal{O}=\{\varnothing\} \cup\left\{t^{\frown} 0\right.$ : $\left.t \in 2^{<\mathbb{N}}\right\}$. Namely, $\mathcal{O}$ is the subset of the Cantor tree consisting of all sequences 
ending with 0 . If $D=\left(s_{t}\right)_{t \in 2^{<\mathbb{N}}}$ is a dyadic subtree of $2^{<\mathbb{N}}$, we let $\mathcal{O}_{D}=\left\{s_{t}: t \in \mathcal{O}\right\}$. Let $h_{D}: \mathcal{O}_{D} \rightarrow \mathbb{N}$ be the unique bijection satisfying $h_{D}\left(s_{t_{1}}\right)<h_{D}\left(s_{t_{2}}\right)$ if either $\left|t_{1}\right|<\left|t_{2}\right|$, or $\left|t_{1}\right|=\left|t_{2}\right|$ and $t_{1} \prec t_{2}$. By $h: \mathcal{O} \rightarrow \mathbb{N}$ we shall denote the bijection corresponding to the Cantor tree itself.

For every $t \in 2^{<\mathbb{N}}$ we let $V_{t}=\left\{\sigma \in 2^{\mathbb{N}}: t \sqsubset \sigma\right\}$; that is, $V_{t}$ is the clopen subset of $2^{\mathbb{N}}$ determined by the node $t$. We set $f_{t}=\chi_{V_{t}}$. Clearly $f_{t} \in C\left(2^{\mathbb{N}}\right)$ and $\left\|f_{t}\right\|=1$. Let $\left(t_{n}\right)$ be the enumeration of the set $\mathcal{O}$ according to the bijection $h$ and consider the corresponding sequence $\left(f_{t_{n}}\right)$. The main properties of the sequence $\left(f_{t_{n}}\right)$ are summarized in the following claim.

Claim 14. The following hold.

(i) The sequence $\left(f_{t_{n}}\right)$ is a normalized monotone basis of $C\left(2^{\mathbb{N}}\right)$.

(ii) Let $D=\left(s_{t}\right)_{t \in 2<\mathbb{N}}$ be a dyadic subtree of $2^{<\mathbb{N}}$ and let $\left(s_{n}\right)$ be the enumeration of the set $\mathcal{O}_{D}$ according to $h_{D}$. Then the corresponding sequence $\left(f_{s_{n}}\right)$ is 1-equivalent to the basis $\left(f_{t_{n}}\right)$.

(iii) For every $t \in 2^{<\mathbb{N}}$ there exists a sequence $\left(w_{n}\right)$ in $2^{<\mathbb{N}}$ with $t \sqsubset w_{n}$ for every $n \in \mathbb{N}$ and such that the sequence $\left(f_{w_{n}}\right)$ is weakly-null.

Proof of Claim 14. We will give the proof of part (i), leaving it to the reader to supply the details for parts (ii) and (iii). So, consider the sequence $\left(f_{t_{n}}\right)$. First we observe that $f_{t} \in \operatorname{span}\left\{f_{t_{n}}: n \in \mathbb{N}\right\}$ for every $t \in 2^{<\mathbb{N}}$. Hence $\overline{\operatorname{span}}\left\{f_{t_{n}}: n \in\right.$ $\mathbb{N}\}=C\left(2^{\mathbb{N}}\right)$. Thus, it is enough to show that $\left(f_{t_{n}}\right)$ is a monotone Schauder basic sequence. To see this, let $k, m \in \mathbb{N}$ with $k<m$ and $a_{0}, \ldots, a_{m} \in \mathbb{R}$. There exists $\sigma \in 2^{\mathbb{N}}$ such that

$$
\left\|\sum_{n=0}^{k} a_{n} f_{t_{n}}\right\|=\left|\sum_{n=0}^{k} a_{n} f_{t_{n}}(\sigma)\right|
$$

We make the following simple (though crucial) observation. Let $l, j \in \mathbb{N}$ with $t_{l} \sqsubset t_{j}$ (by the properties of $h$ this implies that $l<j$ ). Then there exists a node $s \in 2^{<\mathbb{N}}$ with $t_{l} \sqsubset s,|s|=\left|t_{j}\right|$ and such that $f_{t_{j}}(x)=0$ for every $x \in V_{s}$. Using this observation we see that there exists $\tau \in 2^{\mathbb{N}}$ such that $f_{t_{n}}(\tau)=f_{t_{n}}(\sigma)$ if $0 \leq n \leq k$ while $f_{t_{n}}(\tau)=0$ if $k<n \leq m$. Hence

$$
\left\|\sum_{n=0}^{k} a_{n} f_{t_{n}}\right\|=\left|\sum_{n=0}^{k} a_{n} f_{t_{n}}(\sigma)\right|=\left|\sum_{n=0}^{m} a_{n} f_{t_{n}}(\tau)\right| \leq\left\|\sum_{n=0}^{m} a_{n} f_{t_{n}}\right\| .
$$

This shows that $\left(f_{t_{n}}\right)$ is a monotone basis of $C\left(2^{\mathbb{N}}\right)$, as desired.

After this preliminary discussion we are ready to proceed to the proof of part (i). Clearly it is enough to show that if the space $X$ contains an isomorphic copy of $C\left(2^{\mathbb{N}}\right)$, then so does $Y$. So, let $Z$ be a subspace of $X$ which is isomorphic to $C\left(2^{\mathbb{N}}\right)$. We fix an isomorphism $T: C\left(2^{\mathbb{N}}\right) \rightarrow Z$ and we set $K=\|T\| \cdot\left\|T^{-1}\right\|$. Also let $Q: X \rightarrow X / Y$ be the natural quotient map. The basic step for constructing a subspace $Y^{\prime}$ of $Y$ which is isomorphic to $C\left(2^{\mathbb{N}}\right)$ is given in the following claim.

Claim 15. Let $\left(z_{n}\right)$ be a normalized weakly-null sequence in $Z$ and let $r>0$ be arbitrary. Then there exist $k \in \mathbb{N}$ and a vector $y \in Y$ such that $\left\|z_{k}-y\right\|<r$.

Proof of Claim 15. Consider the sequence $\left(Q\left(z_{n}\right)\right)$. By our assumptions, it is weakly-null. The space $X / Y$ has the Schur property. Hence, $\lim _{n}\left\|Q\left(z_{n}\right)\right\|=0$. Let $k \in \mathbb{N}$ with $\left\|Q\left(z_{k}\right)\right\|<r$. By definition, there exists a vector $y \in Y$ such that $\left\|Q\left(z_{k}\right)\right\| \leq\left\|z_{k}-y\right\|<r$. The claim is proved. 
Using Claim 14(iii) and Claim 15, we may construct recursively a dyadic subtree $D=\left(s_{t}\right)_{t \in 2<\mathbb{N}}$ of $2^{<\mathbb{N}}$ and a family $\left(y_{t}\right)_{t \in 2<\mathbb{N}}$ in $Y$ such that, setting $z_{t}=\frac{T\left(f_{s_{t}}\right)}{\left\|T\left(f_{s_{t}}\right)\right\|}$ for every $t \in 2^{<\mathbb{N}}$, we have

$$
\sum_{t \in 2^{<N}}\left\|z_{t}-y_{t}\right\|<\frac{1}{2 K}
$$

By [LT, Proposition 1.a.9] and Claim[14(ii), we see that if $\left(t_{n}\right)$ is the enumeration of the set $\mathcal{O}$ according to $h$, then the corresponding sequence $\left(y_{t_{n}}\right)$ is equivalent to the sequence $\left(f_{t_{n}}\right)$. By Claim[14(i), it follows that the subspace $Y^{\prime}=\overline{\operatorname{span}}\left\{y_{t_{n}}: n \in \mathbb{N}\right\}$ of $Y$ is isomorphic to $C\left(2^{\mathbb{N}}\right)$. The proof of part (i) is completed.

(ii) We argue by contradiction. So, assume that there exists a subspace $Z$ of $X$ which is isomorphic to $E$. As in part (i), let us denote by $Q: X \rightarrow X / Y$ the natural quotient map. The fact that the space $E$ does not contain $\ell_{1}$ yields that the operator $\left.Q\right|_{Z}$ is strictly singular. This, in turn, implies that

$$
\operatorname{dist}\left(S_{Z^{\prime}}, S_{Y}\right)=\min \left\{\|z-y\|: z \in Z^{\prime}, y \in Y \text { and }\|z\|=\|y\|=1\right\}=0
$$

for every infinite-dimensional subspace $Z^{\prime}$ of $Z$. Hence, there exist a subspace $Z^{\prime \prime}$ of $Z$ and a subspace $Y^{\prime}$ of $Y$ which are isomorphic. As $E$ is minimal, we see that $Z^{\prime \prime}$ must contain an isomorphic copy of $E$. Hence so does $Y$, a contradiction. The proof is completed.

\section{Parameterizing the Bourgain-Pisier construction}

In $[\mathrm{BP}$, J. Bourgain and G. Pisier proved the following.

Theorem 16 ([BP, Theorem 2.1). Let $\lambda>1$ and let $X$ be any separable Banach space. Then there exists a separable $\mathcal{L}_{\infty, \lambda+}$-space, denoted by $\mathcal{L}_{\lambda}[X]$, which contains $X$ isometrically and is such that the quotient $\mathcal{L}_{\lambda}[X] / X$ has the Radon-Nikodym and the Schur properties.

This section is devoted to the proof of the following parameterized version of their result.

Theorem 17. For every $\lambda>1$ the set $\mathcal{L}_{\lambda} \subseteq \mathrm{SB} \times \mathrm{SB}$ defined by

$$
(X, Y) \in \mathcal{L}_{\lambda} \Leftrightarrow Y \text { is isometric to } \mathcal{L}_{\lambda}[X]
$$

is analytic.

The section is organized as follows. In $\S 4.1$ we present some preliminary tools needed in the proof of Theorem 17 and the Bourgain-Pisier construction. The construction itself is briefly recalled in $\S 4.2$. The proof of Theorem 17 is given in $\S 4.3$ while in $\S 4.4$ we isolate some of its consequences.

4.1. Preliminary tools. A system of isometric embeddings is a sequence $\left(X_{n}, j_{n}\right)$ where $\left(X_{n}\right)$ is a sequence of Banach spaces and $j_{n}: X_{n} \rightarrow X_{n+1}$ is an isometric embedding for every $n \in \mathbb{N}$. Let us recall the definition of the inductive limit $X$ of a system $\left(X_{n}, j_{n}\right)$ of isometric embeddings. We consider, first, the vector subspace of $\Pi_{n} X_{n}$ consisting of all sequences $\left(x_{n}\right)$ such that $j_{n}\left(x_{n}\right)=x_{n+1}$ for all $n$ large enough. We equip this subspace with the semi-norm $\left\|\left(x_{n}\right)\right\|=\lim _{n}\left\|x_{n}\right\|$. Let $\mathcal{X}$ be the vector space obtained after passing to the quotient by the kernel of that semi-norm. The space $X$ is then defined to be the completion of $\mathcal{X}$. Notice that there exists a sequence $\left(J_{n}\right)$ of isometric embeddings $J_{n}: X_{n} \rightarrow X$ such that 
$J_{n+1} \circ j_{n}=J_{n}$ for every $n \in \mathbb{N}$ and if $E_{n}=J_{n}\left(X_{n}\right)$, then the union $\bigcup_{n} E_{n}$ is dense in $X$. Hence, in practice, we may do as if the sequence $\left(X_{n}\right)$ was an increasing (with respect to inclusion) sequence of subspaces of a larger space, and we may identify the space $X$ with the closure of the vector space $\bigcup_{n} X_{n}$.

We also recall the following construction, due to S. V. Kisliakov Ki].

Definition 18. Let $B$ and $X$ be Banach spaces and $\eta \leq 1$. Also let $S$ be a subspace of $B$ and $u: S \rightarrow X$ a linear operator with $\|u\| \leq \eta$. Let $B \oplus_{1} X$ be the vector space $B \times X$ equipped with the norm $\|(b, x)\|=\|b\|+\|x\|$ and consider the subspace $N=\{(s,-u(s)): s \in S\}$ of $B \oplus_{1} X$. We define $X_{1}=\left(B \oplus_{1} X\right) / N$. Moreover, denoting by $Q: B \oplus_{1} X \rightarrow X_{1}$ the natural quotient map, we define $\tilde{u}: B \rightarrow X_{1}$ and $j: X \rightarrow X_{1}$ by

$$
\tilde{u}(b)=Q((b, 0)) \text { and } j(x)=Q((0, x))
$$

for every $b \in B$ and every $x \in X$. We call the family $\left(X_{1}, j, \tilde{u}\right)$ the canonical triple associated to $(B, S, u, X, \eta)$.

We gather below some basic properties of the canonical triple.

Proposition 19. Let $B, S, u, X$ and $\eta$ be as in Definition 18 and consider the canonical triple $\left(X_{1}, j, \tilde{u}\right)$ associated to $(B, S, u, X, \eta)$. Then $j$ is an isometric embedding, $\|\tilde{u}\| \leq 1$ and $\tilde{u}(s)=j(u(s))$ for every $s \in S$. Moreover, the spaces $B / S$ and $X_{1} / X$ are isometric.

We refer the reader to [Ki] and [Pi] for a proof of Proposition [19] as well as for refinements of it.

We recall two more properties of the above construction which were isolated in [BP, Proposition 1.3]. The first property is its minimality. Specifically, let $B, S, u, X$ and $\eta$ be as in Definition 18 and consider any commutative diagram

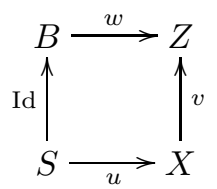

where $Z$ is a Banach space and $w: B \rightarrow Z$ and $v: X \rightarrow Z$ are bounded linear operators. Then there exists a unique bounded operator $T: X_{1} \rightarrow Z$ such that $w(b)=T(\tilde{u}(b))$ for every $b \in B$ and $v(x)=T(j(x))$ for every $x \in X$.

The second one is its uniqueness. Suppose that $\left(X_{1}^{\prime}, j^{\prime}, \tilde{u}^{\prime}\right)$ is another triple satisfying the conclusion of Proposition 19 and the minimality property described above. Then there exists an isometry $T: X_{1} \rightarrow X_{1}^{\prime}$ such that $T(j(x))=j^{\prime}(x)$ for every $x \in X$.

Following $\mathrm{BP}$, we call such a triple $\left(X_{1}, j, \tilde{u}\right)$ as described above, a triple associated to $(B, S, u, X, \eta)$ and we say that the corresponding isometric embedding $j: X \rightarrow X_{1}$ is an $\eta$-admissible embedding.

The basic tool for establishing the crucial properties of the Bourgain-Pisier construction is given in the following theorem.

Theorem 20 ( $\left[\mathrm{BP}\right.$, Theorem 1.6). Let $\eta$ be such that $0<\eta<1$. Also let $\left(F_{n}, j_{n}\right)$ be a system of isometric embeddings, where the sequence $\left(F_{n}\right)$ consists of finitedimensional Banach spaces and for every $n \in \mathbb{N}$ the isometric embedding $j_{n}$ : $F_{n} \rightarrow F_{n+1}$ is $\eta$-admissible. Then the inductive limit of the system $\left(F_{n}, j_{n}\right)$ has the Radon-Nikodym and the Schur properties. 
Remark 1 ( $\mathrm{BP}$, Remark 1.5). Let $B, S, u, X$ and $\eta$ be as in Definition 18 and assume that there exists $0<\delta \leq 1$ such that $\|u(s)\| \geq \delta\|s\|$ for every $s \in S$. Then, for every triple $\left(X_{1}^{\prime}, j^{\prime}, \tilde{u}^{\prime}\right)$ associated to $(B, S, u, X, \eta)$ we have $\left\|\tilde{u}^{\prime}(b)\right\| \geq \delta\|b\|$ for every $b \in B$. Indeed, notice that it is enough to verify this property only for the canonical triple $\left(X_{1}, j, \tilde{u}\right)$. Invoking Definition 18 we see that

$$
\|\tilde{u}(b)\| \stackrel{\text { def }}{=} \inf \{\|b+s\|+\|-u(s)\|: s \in S\} \geq \inf \{\delta\|b+s\|+\delta\|s\|: s \in S\}=\delta\|b\|
$$

for every $b \in B$, as desired.

4.2. The construction of the space $\mathcal{L}_{\lambda}[X]$. In this subsection we shall describe the Bourgain-Pisier construction, following the presentation in $[\mathrm{BP}$. So, let $\lambda>1$ and let $X$ be a separable Banach space. In the argument below we shall use the following simple fact.

Fact 21. Let $H$ be a finite-dimensional space. Also let $\varepsilon>0$ be arbitrary. Then, there exist $m \in \mathbb{N}$, a subspace $S$ of $\ell_{\infty}^{m}$ and an isomorphism $T: S \rightarrow H$ satisfying $\|s\| \leq\|T(s)\| \leq(1+\varepsilon)\|s\|$ for every $s \in S$.

We fix $0<\eta<1$ such that $\frac{1}{\lambda}<\eta<1$. We also fix $\varepsilon>0$ with $1+\varepsilon<\lambda \eta$. Let $\left(F_{n}\right)$ be an increasing sequence of finite-dimensional subspaces of $X$ such that $\bigcup_{n} F_{n}$ is dense in $X$ (the sequence $\left(F_{n}\right)$ is not necessarily strictly increasing, as we are not assuming that the space $X$ is infinite-dimensional). By recursion, we shall construct

(C1) a system $\left(E_{n}, j_{n}\right)$ of isometric embeddings, and

$(\mathrm{C} 2)$ a sequence $\left(G_{n}\right)$ of finite-dimensional spaces

such that for every $n \in \mathbb{N}$ the following are satisfied.

(P1) $G_{n} \subseteq E_{n}$ and $G_{0}=\{0\}$.

(P2) The embedding $j_{n}: E_{n} \rightarrow E_{n+1}$ is $\eta$-admissible and $E_{0}=X$.

(P3) $\left(j_{n-1} \circ \ldots \circ j_{0}\right)\left(F_{n-1}\right) \cup j_{n-1}\left(G_{n-1}\right) \subseteq G_{n}$ for every $n \geq 1$.

(P4) $d\left(G_{n}, \ell_{\infty}^{m_{n}}\right) \leq \lambda$, where $\operatorname{dim} G_{n}=m_{n} \geq n$.

As the first step is identical to the general one, we may assume that for some $k \in \mathbb{N}$ with $k \geq 1$ the spaces $\left(G_{n}\right)_{n=0}^{k}$ and $\left(E_{n}\right)_{n=0}^{k}$ and the $\eta$-admissible isometric embeddings $\left(j_{n}\right)_{n=0}^{k-1}$ have been constructed. Let $H_{k}$ be the subspace of $E_{k}$ spanned by $\left(j_{k-1} \circ \ldots \circ j_{0}\right)\left(F_{k}\right) \cup G_{k}$ (if $k=0$, then we take $\left.H_{0}=F_{0}\right)$. Let $m_{k}$ be the least integer with $m_{k} \geq k+1$ and for which there exist a subspace $S_{k}$ of $\ell_{\infty}^{m_{k}}$ and an isomorphism $T: S_{k} \rightarrow H_{k}$ satisfying $\|s\| \leq\|T(s)\| \leq(1+\varepsilon)\|s\|$ for every $s \in S_{k}$. By Fact 21. $m_{k}$ is well-defined. Define $u: S_{k} \rightarrow E_{k}$ by $u(s)=\frac{1}{\lambda} T(s)$ and notice that $u\left(S_{k}\right)=H_{k},\|u\| \leq \eta$ and $\left\|\left.u^{-1}\right|_{H_{k}}\right\| \leq \lambda$. Let $(Y, j, \tilde{u})$ be the canonical triple associated to $\left(\ell_{\infty}^{m_{k}}, S_{k}, u, E_{k}, \eta\right)$. We set $E_{k+1}=Y, j_{k+1}=j$ and $G_{k+1}=\tilde{u}\left(\ell_{\infty}^{m_{k}}\right)$. By Proposition 19 and Remark 11, the spaces $G_{k+1}$ and $E_{k+1}$, and the embedding $j_{k+1}$ satisfy $(\mathrm{P} 1)-(\mathrm{P} 4)$ above. The construction is completed.

Now let $Z$ be the inductive limit of the system $\left(E_{n}, j_{n}\right)$. As we have remarked in $\S 4.1$, the sequence $\left(E_{n}\right)$ can be identified with an increasing sequence of subspaces of $Z$. Under this point of view, we let $\mathcal{L}_{\lambda}[X]$ be the closure of $\bigcup_{n} G_{n}$. By property (P3), we see that $\mathcal{L}_{\lambda}[X]$ contains an isometric copy of $X$, while by property (P4) it follows that the space $\mathcal{L}_{\lambda}[X]$ is $\mathcal{L}_{\infty, \lambda+}$. Finally, the fact that the quotient $\mathcal{L}_{\lambda}[X] / X$ has the Radon-Nikodym and the Schur properties is essentially a consequence of Theorem 20 (see [BP] for more details). 
4.3. Proof of Theorem 17, Let $\lambda>1$ be given and fix $\eta>0$ and $\varepsilon>0$ such that $\frac{1}{\lambda}<\eta<1$ and $1+\varepsilon<\lambda \eta$. Below we will adopt the following notational conventions. By $\Omega$ we shall denote the Borel subset of $\mathrm{SB} \times \mathrm{SB} \times C\left(2^{\mathbb{N}}\right)^{\mathbb{N}} \times C\left(2^{\mathbb{N}}\right)^{\mathbb{N}}$ defined by

$$
\begin{aligned}
\left(X, Y,\left(x_{n}\right),\left(y_{n}\right)\right) \in \Omega \Leftrightarrow & \forall n \in \mathbb{N}\left(x_{n} \in X \text { and } y_{n} \in Y\right) \text { and } \\
& \left(x_{n}\right) \text { is dense in } X,\left(y_{n}\right) \text { is dense in } Y, \\
& Y \subseteq X \text { and } \forall n \in \mathbb{N} \exists m \in \mathbb{N} \text { with } y_{n}=x_{m} .
\end{aligned}
$$

That is, an element $\left(X, Y,\left(x_{n}\right),\left(y_{n}\right)\right) \in \Omega$ codes a separable Banach space $X$, a dense sequence $\left(x_{n}\right)$ in $X$, a subspace $Y$ of $X$ and a subsequence $\left(y_{n}\right)$ of $\left(x_{n}\right)$ which is dense in $Y$. Given $\omega=\left(X, Y,\left(x_{n}\right),\left(y_{n}\right)\right) \in \Omega$ we set $p_{0}(\omega)=X$ and $p_{1}(\omega)=Y$. We will reserve the letter $t$ to denote elements of $\Omega^{<\mathbb{N}}$. The letter $\alpha$ shall be used to denote elements of $\Omega^{\mathbb{N}}$. For every $t \in \Omega^{<\mathbb{N}}$ non-empty and every $i<|t|$ we set $X_{i}^{t}=p_{0}(t(i))$ and $Y_{i}^{t}=p_{1}(t(i))$. Respectively, for every $\alpha \in \Omega^{\mathbb{N}}$ and every $i \in \mathbb{N}$ we set $X_{i}^{\alpha}=p_{0}(\alpha(i))$ and $Y_{i}^{\alpha}=p_{1}(\alpha(i))$. If $X, Y$ and $Z$ are non-empty sets and $f: X \times Y \rightarrow Z$ is a map, then for every $x \in X$ by $f^{x}$ we shall denote the function $f^{x}: Y \rightarrow Z$ defined by $f^{x}(y)=f(x, y)$ for every $y \in Y$. Finally, by $d_{m}: \mathrm{SB} \rightarrow C\left(2^{\mathbb{N}}\right)(m \in \mathbb{N})$ we denote the sequence of Borel maps obtained by Proposition 11 applied for $X=C\left(2^{\mathbb{N}}\right)$.

The proof of Theorem 17 is based on the fact that we can appropriately encode the Bourgain-Pisier construction so that it can be performed "uniformly" in $X$. To this end, we introduce the following terminology.

(A) Let $k \in \mathbb{N}$ with $k \geq 2$. A code of length $k$ is a pair $(C, \phi)$ where $C$ is a Borel subset of $\Omega^{k}$ and $\phi: C \times C\left(2^{\mathbb{N}}\right) \rightarrow C\left(2^{\mathbb{N}}\right)$ is a Borel map such that for every $t \in C$ the following are satisfied.

(C1) For every $i<k$ the space $Y_{i}^{t}$ is finite-dimensional and $Y_{0}^{t}=\{0\}$.

(C2) The map $\phi^{t}: X_{k-2}^{t} \rightarrow C\left(2^{\mathbb{N}}\right)$ is a linear isometric embedding satisfying $\phi^{t}\left(X_{k-2}^{t}\right) \subseteq X_{k-1}^{t}$ and $\phi^{t}\left(Y_{k-2}^{t}\right) \subseteq Y_{k-1}^{t}$.

The code of length 1 is the pair $\left(C_{1}, \phi_{1}\right)$ where $C_{1} \subseteq \Omega$ and $\phi_{1}: C_{1} \times C\left(2^{\mathbb{N}}\right) \rightarrow C\left(2^{\mathbb{N}}\right)$ are defined by

$$
t=\left(X, Y,\left(x_{n}\right),\left(y_{n}\right)\right) \in C_{1} \Leftrightarrow Y=\{0\}
$$

and $\phi_{1}(t, x)=x$ for every $t \in C_{1}$ and every $x \in C\left(2^{\mathbb{N}}\right)$. Clearly $C_{1}$ is Borel and $\phi_{1}$ is a Borel map. Notice that for every $X \in \mathrm{SB}$ there exists $t \in C_{1}$ with $X=X_{0}^{t}$.

(B) Let $\left\{\left(C_{k}, \phi_{k}\right): k \geq 1\right\}$ be a sequence such that for every $k \geq 1$ the pair $\left(C_{k}, \phi_{k}\right)$ is a code of length $k$. We say that the sequence $\left\{\left(C_{k}, \phi_{k}\right): k \geq 1\right\}$ is a tree-code if for every $k, m \in \mathbb{N}$ with $1 \leq k \leq m$ we have $C_{k}=\left\{t \mid k: t \in C_{m}\right\}$. The body $\mathcal{C}$ of a tree-code $\left\{\left(C_{k}, \phi_{k}\right): k \geq 1\right\}$ is defined by $\mathcal{C}=\left\{\alpha \in \Omega^{\mathbb{N}}: \alpha \mid k \in C_{k} \forall k \geq 1\right\}$. Clearly $\mathcal{C}$ is a Borel subset of $\Omega^{\mathbb{N}}$.

Let $\left\{\left(C_{k}, \phi_{k}\right): k \geq 1\right\}$ be a tree-code and let $\mathcal{C}$ be its body. For every $k \geq 1$, the map $\phi_{k}$ induces a map $\Phi_{k}: \mathcal{C} \times C\left(2^{\mathbb{N}}\right) \rightarrow C\left(2^{\mathbb{N}}\right)$ defined by $\Phi_{k}(\alpha, x)=\phi_{k}(\alpha \mid k, x)$ for every $\alpha \in \mathcal{C}$ and every $x \in C\left(2^{\mathbb{N}}\right)$. We need to introduce two more maps. First, for every $n, m \in \mathbb{N}$ with $n<m$ we define $\Phi_{n, m}: \mathcal{C} \times C\left(2^{\mathbb{N}}\right) \rightarrow C\left(2^{\mathbb{N}}\right)$ recursively by the rules $\Phi_{n, n+1}(\alpha, x)=\Phi_{n+2}(\alpha, x)$ and $\Phi_{n, m+1}(\alpha, x)=\Phi_{m+2}\left(\alpha, \Phi_{n, m}(\alpha, x)\right)$. We also set $J_{n}=\Phi_{n+2}$ for every $n \in \mathbb{N}$. We isolate, for future use, the following fact concerning these maps. Its proof is a straightforward consequence of the relevant definitions and of condition $(\mathrm{C} 2)$. 
Fact 22. Let $\left\{\left(C_{k}, \phi_{k}\right): k \geq 1\right\}$ be a tree-code and let $\mathcal{C}$ be its body. Then the following are satisfied.

(i) For every $n, k, m \in \mathbb{N}$ with $k \geq 1$ and $n<m$, the maps $\Phi_{k}$ and $\Phi_{n, m}$ are Borel. Moreover, for every $\alpha \in \mathcal{C}$ we have $\Phi_{n, m}^{\alpha}\left(X_{n}^{\alpha}\right) \subseteq X_{m}^{\alpha}$ and $\Phi_{n, m}^{\alpha}\left(Y_{n}^{\alpha}\right) \subseteq Y_{m}^{\alpha}$.

(ii) Let $\alpha \in \mathcal{C}$. Then, for every $n \in \mathbb{N}$ the map $\left.J_{n}^{\alpha}\right|_{X_{n}^{\alpha}}$ is a linear isometric embedding satisfying $J_{n}^{\alpha}\left(X_{n}^{\alpha}\right) \subseteq X_{n+1}^{\alpha}$ and $J_{n}^{\alpha}\left(Y_{n}^{\alpha}\right) \subseteq Y_{n+1}^{\alpha}$.

(C) Let $\left\{\left(C_{k}, \phi_{k}\right): k \geq 1\right\}$ be a tree-code and let $\mathcal{C}$ be its body. Let $\alpha \in \mathcal{C}$. Consider the sequence $\left(X_{0}^{\alpha}, Y_{0}^{\alpha}, X_{1}^{\alpha}, Y_{1}^{\alpha}, \ldots\right)$ and notice that $Y_{n}^{\alpha}$ is a finite-dimensional subspace of $X_{n}^{\alpha}$ for every $n \in \mathbb{N}$. In the coding we are developing, the sequences $\left(X_{n}^{\alpha}\right)$ and $\left(Y_{n}^{\alpha}\right)$ will correspond to the sequences $\left(E_{n}\right)$ and $\left(G_{n}\right)$ obtained following the Bourgain-Pisier construction performed on the space $X=X_{0}^{\alpha}$. This is made precise using the auxiliary concept of $\lambda$-coherence, which we are about to introduce.

So, let $\alpha \in \mathcal{C}$ be arbitrary. For every $n \in \mathbb{N}$, let $F_{n}\left(X_{0}^{\alpha}\right)=\overline{\operatorname{span}}\left\{d_{i}\left(X_{0}^{\alpha}\right): i \leq n\right\}$. Clearly $\left(F_{n}\left(X_{0}^{\alpha}\right)\right)$ is an increasing sequence of finite-dimensional subspaces of $X_{0}^{\alpha}$ with $\bigcup_{n} F_{n}\left(X_{0}^{\alpha}\right)$ dense in $X_{0}^{\alpha}$. Let $\left(E_{n}^{\alpha}, j_{n}^{\alpha}\right)$ be the system of isometric embeddings and $\left(G_{n}^{\alpha}\right)$ be the sequence of finite-dimensional spaces obtained by performing the construction described in $\S 4.2$ to the space $X_{0}^{\alpha}$, the sequence $\left(F_{n}\left(X_{0}^{\alpha}\right)\right)$ and the numerical parameters $\lambda, \eta$ and $\varepsilon$. We will say that the tree-code $\left\{\left(C_{k}, \phi_{k}\right): k \geq 1\right\}$ is $\lambda$-coherent if for every $\alpha \in \mathcal{C}$ there exists a sequence $T_{n}^{\alpha}: X_{n}^{\alpha} \rightarrow E_{n}^{\alpha}(n \geq 1)$ of isometries such that $G_{n}^{\alpha}=T_{n}^{\alpha}\left(Y_{n}^{\alpha}\right)$ for every $n \geq 1$ and making the following diagram commutative:

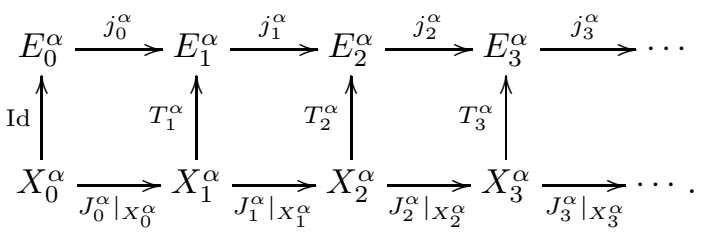

The basic property guaranteed by the above requirements is isolated in the following fact (the proof is straightforward).

Fact 23. Let $\left\{\left(C_{k}, \phi_{k}\right): k \geq 1\right\}$ be a $\lambda$-coherent tree-code and let $\mathcal{C}$ be its body. Also let $\alpha \in \mathcal{C}$. Then the inductive limit of the system of embeddings $\left(Y_{n}^{\alpha},\left.J_{n}^{\alpha}\right|_{Y_{n}^{\alpha}}\right)$ is isometric to the space $\mathcal{L}_{\lambda}\left[X_{0}^{\alpha}\right]$.

We are ready to state the main technical step towards the proof of Theorem 17

Lemma 24. There exists a $\lambda$-coherent tree-code $\left\{\left(C_{k}, \phi_{k}\right): k \geq 1\right\}$.

Granting Lemma 24, the proof is completed as follows. Let $\left\{\left(C_{k}, \phi_{k}\right): k \geq 1\right\}$ be the $\lambda$-coherent tree-code obtained above. Denote by $\mathcal{C}$ its body. By Fact 23 , we have

$$
\begin{aligned}
&(X, Y) \in \mathcal{L}_{\lambda} \Leftrightarrow \quad \exists \alpha \in \Omega^{\mathbb{N}} \text { with } \alpha \in \mathcal{C}, X=X_{0}^{\alpha} \text { and such that } Y \text { is isometric } \\
& \text { to the inductive limit of the system }\left(Y_{n}^{\alpha},\left.J_{n}^{\alpha}\right|_{Y_{n}^{\alpha}}\right) .
\end{aligned}
$$

Let $\alpha \in \mathcal{C}$. There is a canonical dense sequence in the inductive limit $Z^{\alpha}$ of the system $\left(Y_{n}^{\alpha},\left.J_{n}^{\alpha}\right|_{Y_{n}^{\alpha}}\right)$. Indeed, by the discussion in $\S 4.1$, the sequence of spaces $\left(Y_{n}^{\alpha}\right)$ can be identified with an increasing sequence of subspaces of $Z^{\alpha}$. Under this point of view, the sequence $\left(d_{m}\left(Y_{n}^{\alpha}\right)\right)(n, m \in \mathbb{N})$ is a dense sequence in $Z^{\alpha}$. Let 
$\left\{\left(n_{i}, m_{i}\right): i \in \mathbb{N}\right\}$ be an enumeration of the set $\mathbb{N} \times \mathbb{N}$ such that $\max \left\{n_{i}, m_{i}\right\} \leq i$ for every $i \in \mathbb{N}$. It follows that

$$
\begin{aligned}
(X, Y) \in \mathcal{L}_{\lambda} \Leftrightarrow & \exists\left(y_{i}\right) \in C\left(2^{\mathbb{N}}\right)^{\mathbb{N}} \exists \alpha \in \Omega^{\mathbb{N}} \text { with } \alpha \in \mathcal{C}, X=X_{0}^{\alpha} \text { and } \\
& Y=\overline{\operatorname{span}}\left\{y_{i}: i \in \mathbb{N}\right\} \text { and } \forall l \in \mathbb{N} \forall b_{0}, \ldots, b_{l} \in \mathbb{Q} \\
& \left\|\sum_{i=0}^{l} b_{i} y_{i}\right\|=\left\|\sum_{i=0}^{l} b_{i} \Phi_{n_{i}, l+1}\left(\alpha, d_{m_{i}}\left(Y_{n_{i}}^{\alpha}\right)\right)\right\| .
\end{aligned}
$$

Invoking Fact 22(i), we get that the above formula gives an analytic definition of the set $\mathcal{L}_{\lambda}$, as desired.

So, it remains to prove Lemma 24. To this end, we need the following easy fact (the proof is left to the interested reader).

Fact 25. Let $S$ be a standard Borel space, $X$ be a Polish space and $f_{n}: S \rightarrow X$ $(n \in \mathbb{N})$ be a sequence of Borel maps. Then, the map $F: S \rightarrow F(X)$, defined by $F(s)=\overline{\left\{f_{n}(s): n \in \mathbb{N}\right\}}$ for every $s \in S$, is Borel.

We are ready to proceed to the proof of Lemma 24,

Proof of Lemma 24. The $\lambda$-coherent tree-code $\left\{\left(C_{k}, \phi_{k}\right): k \geq 1\right\}$ will be constructed by recursion. For $k=1$ we let $\left(C_{1}, \phi_{1}\right)$ be the code of length 1 defined in (A) above. Assume that for some $k \geq 1$ and every $l \leq k$ we have constructed the code $\left(C_{l}, \phi_{l}\right)$ of length $l$. We will construct the code $\left(C_{k+1}, \phi_{k+1}\right)$ of length $k+1$.

First, we define recursively a family of Borel functions $f_{l}: C_{k} \times C\left(2^{\mathbb{N}}\right) \rightarrow C\left(2^{\mathbb{N}}\right)$ $(1 \leq l \leq k)$ by the rule $f_{1}(t, x)=x$ and $f_{l+1}(t, x)=\phi_{l+1}\left(t \mid l+1, f_{l}(t, x)\right)$. Notice that $f_{k}^{t}\left(X_{0}^{t}\right) \subseteq X_{k-1}^{t}$ for every $t \in C_{k}$. Also let $F_{k-1}: \mathrm{SB} \rightarrow \mathrm{SB}$ and $H_{k}: C_{k} \rightarrow \mathrm{SB}$ be defined by $F_{k-1}(X)=\overline{\operatorname{span}}\left\{d_{i}(X): i \leq k-1\right\}$ and

$$
H_{k}(t)=\overline{\operatorname{span}}\left\{Y_{k-1}^{t} \cup f_{k}^{t}\left(F_{k-1}\left(X_{0}^{t}\right)\right)\right\}
$$

respectively. Observe that for every $X \in \mathrm{SB}$ and every $t \in C_{k}$ the spaces $F_{k-1}(X)$ and $H_{k}(t)$ are both finite-dimensional subspaces of $X$ and $X_{k-1}^{t}$ respectively.

Claim 26. The maps $F_{k-1}$ and $H_{k}$ are Borel.

Proof of Claim 26. For every $s \in \mathbb{Q}^{k}$ consider the map $f_{s}: \mathrm{SB} \rightarrow C\left(2^{\mathbb{N}}\right)$ defined by $f_{s}(X)=\sum_{i=0}^{k-1} s(i) d_{i}(X)$. Clearly $f_{s}$ is Borel. Notice that $F_{k-1}(X)$ is equal to the closure of the set $\left\{f_{s}(X): s \in \mathbb{Q}^{k}\right\}$. Invoking Fact 25, the Borelness of the map $F_{k-1}$ follows. The proof that $H_{k}$ is also Borel proceeds similarly. The claim is proved.

We fix a dense sequence $\left(\sigma_{i}\right)$ in $2^{\mathbb{N}}$. For every $d \in \mathbb{N}$ with $d \geq k$ we define an operator $v_{d}: C\left(2^{\mathbb{N}}\right) \rightarrow \ell_{\infty}^{d}$ by

$$
v_{d}(f)=\left(f\left(\sigma_{0}\right), \ldots, f\left(\sigma_{d-1}\right)\right) .
$$

Notice that $\left\|v_{d}(f)\right\| \leq\|f\|$. Moreover, observe that the map $C\left(2^{\mathbb{N}}\right) \ni f \mapsto\left\|v_{d}(f)\right\|$ is continuous. For every $d \geq k$ let $B_{d}$ be the subset of $C_{k}$ defined by

$$
\begin{aligned}
t \in B_{d} & \Leftrightarrow \forall f \in H_{k}(t) \text { we have }\|f\| \leq(1+\varepsilon)\left\|v_{d}(f)\right\| \\
& \Leftrightarrow \forall n \in \mathbb{N} \text { we have }\left\|d_{n}\left(H_{k}(t)\right)\right\| \leq(1+\varepsilon)\left\|v_{d}\left(d_{n}\left(H_{k}(t)\right)\right)\right\| .
\end{aligned}
$$

By the above formula, we see that $B_{d}$ is Borel. Also observe that $C_{k}=\bigcup_{d \geq k} B_{d}$. We define recursively a partition $\left\{P_{d}: d \geq k\right\}$ of $C_{k}$ by the rule $P_{k}=B_{k}$ and $P_{d+1}=B_{d+1} \backslash\left(P_{k} \cup \ldots \cup P_{d}\right)$. Notice that $P_{d}$ is a Borel subset of $B_{d}$. 
Let $d \geq k$ be arbitrary. By $Z$ we shall denote the vector space $C\left(2^{\mathbb{N}}\right) \times \ell_{\infty}^{d}$ equipped with the norm $\|(f, a)\|=\|f\|+\|a\|$ for every $f \in C\left(2^{\mathbb{N}}\right)$ and every $a \in \ell_{\infty}^{d}$. Consider the map $N: P_{d} \rightarrow \operatorname{Subs}(Z)$ defined by

$$
N(t)=\left\{\left(-f, \lambda v_{d}(f)\right): f \in H_{k}(t)\right\} .
$$

Arguing as in Claim 26 it is easy to see that $N$ is Borel. Let $\mathbf{d}_{m}: \operatorname{Subs}(Z) \rightarrow Z$ $(m \in \mathbb{N})$ be the sequence of Borel maps obtained by Proposition 11 applied for $X=$ $Z$. We recall that by $d_{m}: C\left(2^{\mathbb{N}}\right) \rightarrow C\left(2^{\mathbb{N}}\right)(m \in \mathbb{N})$ we denote the corresponding sequence obtained for $X=C\left(2^{\mathbb{N}}\right)$. We may, and we will, assume that $d_{0}(X)=0$ and $\mathbf{d}_{0}\left(Z^{\prime}\right)=0$ for every $X \in \mathrm{SB}$ and every $Z^{\prime} \in \operatorname{Subs}(Z)$. We also fix a countable dense subset $\left(r_{m}\right)$ of $\ell_{\infty}^{d}$ such that $r_{0}=0$. Let $Q: P_{d} \times Z \rightarrow \mathbb{R}$ be the map

$$
Q(t, z)=\inf \left\{\left\|z+\mathbf{d}_{m}(N(t))\right\|: m \in \mathbb{N}\right\} .
$$

Clearly $Q$ is Borel. We fix a bijection $\langle\cdot, \cdot\rangle: \mathbb{N} \times \mathbb{N} \rightarrow \mathbb{N}$. For every $n \in \mathbb{N}$ by $m_{n}^{0}$ and $m_{n}^{1}$ we shall denote the unique integers satisfying $n=\left\langle m_{n}^{0}, m_{n}^{1}\right\rangle$. We define $C_{d} \subseteq \Omega^{k+1}$ by

$$
\begin{aligned}
& t^{\prime} \in C_{d} \Leftrightarrow \quad t^{\prime} \mid k \in P_{d} \text { and if } t^{\prime}(k)=\left(X, Y,\left(x_{n}\right),\left(y_{n}\right)\right) \text { and } t=t^{\prime} \mid k, \\
& \text { then } \forall i \in \mathbb{N} \forall b_{0}, \ldots, b_{i} \in \mathbb{Q} \text { we have } \\
&\left\|\sum_{n=0}^{i} b_{n} x_{n}\right\|=Q\left(t, \sum_{n=0}^{i} b_{n}\left(d_{m_{n}^{0}}\left(X_{k-1}^{t}\right), r_{m_{n}^{1}}\right)\right) \text { and } \\
& \forall n \in \mathbb{N} \text { we have } y_{n}=x_{\langle 0, n\rangle} .
\end{aligned}
$$

Clearly the above formula defines a Borel subset of $\Omega^{k+1}$. Also observe that $C_{d} \cap$ $C_{d^{\prime}}=\varnothing$ if $d \neq d^{\prime}$.

Let us comment on some properties of the set $C_{d}$. Fix $t^{\prime} \in C_{d}$ and set $t=t^{\prime} \mid k$. By definition, we have $t \in P_{d} \subseteq B_{d}$. It follows that the operator $v_{d}: H_{k}(t) \rightarrow \ell_{\infty}^{d}$ is an isomorphic embedding satisfying $\left\|v_{d}(x)\right\| \leq\|x\| \leq(1+\varepsilon)\left\|v_{d}(x)\right\|$ for every $x \in H_{k}(t)$. We set $S_{t}=v_{d}\left(H_{k}(t)\right)$ and we define $u: S_{t} \rightarrow X_{k-1}^{t}$ by

$$
u(s)=\frac{1}{\lambda}\left(\left.v_{d}\right|_{H_{k}(t)}\right)^{-1}(s) .
$$

By the choice of $\varepsilon$ and $\lambda$, we see that $\|u\| \leq \eta$. Let $\left(Z_{t}, j, \tilde{u}\right)$ be the canonical triple associated to $\left(\ell_{\infty}^{d}, S_{t}, u, X_{k-1}^{t}, \eta\right)$. There is a natural way to select a dense sequence in $Z_{t}$. Indeed, as in Definition[18, consider the Banach space $\ell_{\infty}^{d} \oplus_{1} X_{k-1}^{t}$ and let $Q_{t}$ : $\ell_{\infty}^{d} \oplus_{1} X_{k-1}^{t} \rightarrow Z_{t}$ be the natural quotient map. Setting $z_{n}=Q_{t}\left(\left(r_{m_{n}^{1}}, d_{m_{n}^{0}}\left(X_{k-1}^{t}\right)\right)\right)$ for every $n \in \mathbb{N}$, we see that the sequence $\left(z_{n}\right)$ is a dense sequence in $Z_{t}$. Let $t^{\prime}(k)=\left(X_{k}^{t^{\prime}}, Y_{k}^{t^{\prime}},\left(x_{n}\right),\left(y_{n}\right)\right)$. By the definition of the set $C_{d}$, it follows that the map

$$
Z_{t} \ni z_{n} \mapsto x_{n} \in X_{k}^{t^{\prime}}
$$

can be extended to a linear isometry $T_{t^{\prime}}: Z_{t} \rightarrow X_{k}^{t^{\prime}}$. In other words, we have the following commutative diagram:

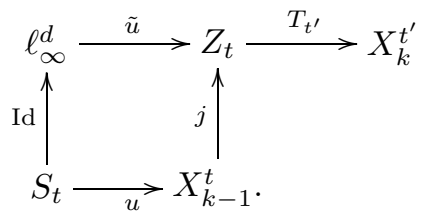


It is clear from what we have said that the map

$$
X_{k-1}^{t} \ni d_{n}\left(X_{k-1}^{t}\right) \mapsto x_{\langle n, 0\rangle} \in X_{k}^{t^{\prime}}
$$

can also be extended to the linear isometric embedding $J^{t^{\prime}}: X_{k-1}^{t} \rightarrow X_{k}^{t^{\prime}}$ satisfying $J^{t^{\prime}}\left(Y_{k-1}^{t}\right) \subseteq Y_{k}^{t^{\prime}}$. Moreover, it is easy to see that this extension can be done "uniformly" in $t^{\prime}$. Precisely, there exists a Borel map $\phi_{d}: C_{d} \times C\left(2^{\mathbb{N}}\right) \rightarrow C\left(2^{\mathbb{N}}\right)$ such that for every $t^{\prime} \in C_{d}$ we have $\phi_{d}\left(t^{\prime}, x\right)=J^{t^{\prime}}(x)$ for every $x \in X_{k-1}^{t^{\prime} \mid k}=X_{k-1}^{t^{\prime}}$.

We are finally in a position to construct the code $\left(C_{k+1}, \phi_{k+1}\right)$ of length $k+1$. First we set

$$
C_{k+1}=\bigcup_{d \geq k} C_{d}
$$

As we have already remarked, the sets $\left(C_{d}\right)_{d \geq k}$ are pairwise disjoint. We define $\phi_{k+1}: C_{k+1} \times C\left(2^{\mathbb{N}}\right) \rightarrow C\left(2^{\mathbb{N}}\right)$ as follows. Let $\left(t^{\prime}, x\right) \in C_{k+1} \times C\left(2^{\mathbb{N}}\right)$ and let $d$ be the unique integer with $t^{\prime} \in C_{d}$. We set $\phi_{k+1}\left(t^{\prime}, x\right)=\phi_{d}\left(t^{\prime}, x\right)$. Clearly, the just defined pair $\left(C_{k+1}, \phi_{k+1}\right)$ is a code of length $k+1$.

This completes the recursive construction of the family $\left\{\left(C_{k}, \phi_{k}\right): k \geq 1\right\}$. That the family $\left\{\left(C_{k}, \phi_{k}\right): k \geq 1\right\}$ is a tree-code follows immediately by the definition of the set $C_{d}$ above. Moreover, as one can easily realize, the tree-code $\left\{\left(C_{k}, \phi_{k}\right): k \geq 1\right\}$ is in addition $\lambda$-coherent, as desired.

As we have already indicated above, having completed the proof of Lemma 24, the proof of Theorem 17 is also completed.

4.4. Consequences. We start with the following.

Corollary 27. Let $A$ be an analytic subset of NU. Then there exists an analytic subset $A^{\prime}$ of $\mathrm{NU}$ with the following properties.

(i) Every $Y \in A^{\prime}$ has a Schauder basis.

(ii) For every $X \in A$ there exists $Y \in A^{\prime}$ containing an isometric copy of $X$.

Proof. Let $\mathcal{L}_{2}$ be the analytic subset of SB $\times \mathrm{SB}$ obtained by Theorem 17 applied for $\lambda=2$. We define $A^{\prime}$ by

$$
Y \in A^{\prime} \Leftrightarrow \exists X \in \mathrm{SB} \text { with } X \in A \text { and }(X, Y) \in \mathcal{L}_{2} .
$$

Clearly $A^{\prime}$ is analytic. By Theorem 9, Proposition 13(i) and Theorem 16 the set $A^{\prime}$ is as desired.

Let $X$ be a non-universal separable Banach space and let $\lambda>1$. By Theorem 16 and Proposition $13(\mathrm{i})$, the space $\mathcal{L}_{\lambda}[X]$ is also non-universal. We have the following quantitative refinement of this fact.

Corollary 28. Let $\lambda>1$. Then there exists a map $f_{\lambda}: \omega_{1} \rightarrow \omega_{1}$ such that for every $\xi<\omega_{1}$ and every separable Banach space $X$ with $\phi_{\mathrm{NU}}(X) \leq \xi$ we have $\phi_{\mathrm{NU}}\left(\mathcal{L}_{\lambda}[X]\right) \leq f_{\lambda}(\xi)$.

In particular, there exists a map $f: \omega_{1} \rightarrow \omega_{1}$ such that for every $\xi<\omega_{1}$, every separable Banach space $X$ with $\phi_{\mathrm{NU}}(X) \leq \xi$ embeds isometrically into a Banach space $Y$ with a Schauder basis satisfying $\phi_{\mathrm{NU}}(Y) \leq f(\xi)$.

Proof. By Theorem 12(i), we know that the map $\phi_{\mathrm{NU}}$ is a $\Pi_{1}^{1}$-rank on NU. Let $\xi<\omega_{1}$ be arbitrary and set

$$
A_{\xi}=\left\{X \in \mathrm{NU}: \phi_{\mathrm{NU}}(X) \leq \xi\right\} .
$$


By Lemma 10(i), the set $A_{\xi}$ is analytic (in fact Borel). Let $\mathcal{L}_{\lambda}$ be the analytic subset of $\mathrm{SB} \times \mathrm{SB}$ obtained by Theorem 17 applied for the given $\lambda$. We define $B_{\xi}$ by

$$
Y \in B_{\xi} \Leftrightarrow \exists X \in \mathrm{SB} \text { with } X \in A_{\xi} \text { and }(X, Y) \in \mathcal{L}_{\lambda} .
$$

As in Corollary 27, we see that $B_{\xi}$ is an analytic subset of NU. By Lemma 10(ii), we have that $\sup \left\{\phi_{\mathrm{NU}}(Y): Y \in B_{\xi}\right\}<\omega_{1}$. We set

$$
f_{\lambda}(\xi)=\sup \left\{\phi_{\mathrm{NU}}(Y): Y \in B_{\xi}\right\} .
$$

Clearly the map $f_{\lambda}$ is as desired.

We close this subsection by presenting the following analogues of Corollaries 27 and 28 for the class $\mathrm{NC}_{Z}$. They are both derived using identical arguments as above.

Corollary 29. Let $Z$ be a minimal Banach space not containing $\ell_{1}$. Also let $A$ be an analytic subset of $\mathrm{NC}_{Z}$. Then there exists an analytic subset $A^{\prime}$ of $\mathrm{NC}_{Z}$ with the following properties.

(i) Every $Y \in A^{\prime}$ has a Schauder basis.

(ii) For every $X \in A$ there exists $Y \in A^{\prime}$ containing an isometric copy of $X$.

Corollary 30. Let $Z$ be a minimal Banach space with a Schauder basis and not containing $\ell_{1}$. Also let $\lambda>1$. Then there exists a map $f_{\lambda}^{Z}: \omega_{1} \rightarrow \omega_{1}$ such that for every $\xi<\omega_{1}$ and every separable Banach space $X$ with $\phi_{\mathrm{NC}_{Z}}(X) \leq \xi$ we have $\phi_{\mathrm{NC}_{Z}}\left(\mathcal{L}_{\lambda}[X]\right) \leq f_{\lambda}^{Z}(\xi)$.

In particular, there exists a map $f_{Z}: \omega_{1} \rightarrow \omega_{1}$ such that for every $\xi<\omega_{1}$, every separable Banach space $X$ with $\phi_{\mathrm{NC}_{Z}}(X) \leq \xi$ embeds isometrically into a Banach space $Y$ with a Schauder basis satisfying $\phi_{\mathrm{NC}_{Z}}(Y) \leq f_{Z}(\xi)$.

\section{The Strong Boundedness OF THE ClAss OF NON-UNIVERSAL SPACES WITH A SCHAUDER BASIS}

In $\mathrm{AD}$ ] it was shown that the class of non-universal spaces with a Schauder basis is strongly bounded. More precisely, the following was proved.

Theorem 31 ( $\mathrm{AD}$, Proposition 83). Let $A$ be an analytic subset of $\mathrm{SB}$ such that every $Y \in A$ is non-universal and has a Schauder basis. Then there exists a non-universal space $Z$, with a Schauder basis, that contains every $Y \in A$ as a complemented subspace.

Our aim in this section is to sketch a proof of Theorem 31 which, although simpler than the one given in $\mathrm{AD}$, still highlights some of the basic ideas developed in that work.

But before that, we need to introduce some pieces of notation. For technical reasons (that will become transparent below), we need to work with trees consisting of non-empty finite sequences. In particular, if $\Lambda$ is a countable set and $T$ is a tree on $\Lambda$, then we denote by $\mathrm{T}$ the set $T \backslash\{\varnothing\}$. Namely, $\mathrm{T}$ consists of all non-empty finite sequences of $T$. We call the set $\mathrm{T}$, for obvious reasons, the tree of non-empty sequences of $T$.

A starting difficulty in the proof of Theorem 31 is how one builds a space out of a class of spaces. The technical (and conceptual) device is provided in the following definition. 
Definition 32 (馬, Definition 13). Let $X$ be a Banach space, $\Lambda$ a countable set and $T$ a pruned tree on $\Lambda$. Also let $\left(x_{t}\right)_{t \in T}$ be a normalized sequence in $X$ indexed by the tree $T$ of non-empty sequences of $T$. We say that $\mathfrak{X}=\left(X, \Lambda, T,\left(x_{t}\right)_{t \in T}\right)$ is a Schauder tree basis if the following are satisfied.

(1) $X=\overline{\operatorname{span}}\left\{x_{t}: t \in T\right\}$.

(2) For every $\sigma \in[T]$ the sequence $\left(x_{\sigma \mid n}\right)_{n \geq 1}$ is a (normalized) bi-monotone Schauder basic sequence.

For every Schauder tree basis $\mathfrak{X}=\left(X, \Lambda, T,\left(x_{t}\right)_{t \in \mathrm{T}}\right)$ and every $\sigma \in[T]$ we let $X_{\sigma}=\overline{\operatorname{span}}\left\{x_{\sigma \mid n}: n \geq 1\right\}$. Notice that in Definition 32 we do not assume that the subspace $X_{\sigma}$ of $X$ is complemented. Notice also that if $\sigma, \tau \in[T]$ with $\sigma \neq \tau$, then this does not necessarily imply that $X_{\sigma} \neq X_{\tau}$. The following lemma reveals the critical role of Schauder tree bases in the construction of universal spaces. It is based on a technique in Descriptive Set Theory, introduced by R. M. Solovay, known as "unfolding".

Lemma 33. Let $A$ be an analytic subset of $\mathrm{SB}$ such that every $Y \in A$ has a Schauder basis. Then there exist a separable Banach space $X$, a pruned tree $T$ on $\mathbb{N} \times \mathbb{N}$ and a normalized sequence $\left(x_{t}\right)_{t \in \mathrm{T}}$ in $X$ such that the following are satisfied.

(i) The family $\mathfrak{X}=\left(X, \mathbb{N} \times \mathbb{N}, T,\left(x_{t}\right)_{t \in \mathrm{T}}\right)$ is a Schauder tree basis.

(ii) For every $Y \in A$ there exists $\sigma \in[T]$ with $Y \cong X_{\sigma}$.

(iii) For every $\sigma \in[T]$ there exists $Y \in A$ with $X_{\sigma} \cong Y$.

Proof. Let $U$ be the universal space of A. Pełczyński for Schauder basic sequences (see $[\mathrm{P}]$ ). The space $U$ has a Schauder basis $\left(u_{n}\right)$ satisfying, among other properties, the following one. For every semi-normalized Schauder basic sequence $\left(x_{n}\right)$ in a Banach space $X$, there exists $L=\left\{l_{0}<l_{1}<\ldots\right\} \in[\mathbb{N}]$ such that $\left(x_{n}\right)$ is equivalent to $\left(u_{l_{n}}\right)$. By passing to an equivalent norm and normalizing if necessary, we may additionally assume the basis $\left(u_{n}\right)$ of $U$ is normalized and bi-monotone. Notice that these properties are inherited by the subsequences of $\left(u_{n}\right)$. For every $L=\left\{l_{0}<l_{1}<\ldots\right\} \in[\mathbb{N}]$ we let $U_{L}=\overline{\operatorname{span}}\left\{u_{l_{n}}: n \in \mathbb{N}\right\}$. By identifying the space $U$ with one of its isometric copies in $C\left(2^{\mathbb{N}}\right)$, we see that the map $\Phi:[\mathbb{N}] \rightarrow \mathrm{SB}$, defined by $\Phi(L)=U_{L}$, is Borel.

Now let $A$ be as in the statement of the lemma and put

$$
A \cong=\{Z \in \mathrm{SB}: \exists Y \in A \text { such that } Z \cong Y\} .
$$

That is, $A \cong$ is the isomorphic saturation of $A$. It is easy to see that the equivalence relation $\cong$ of isomorphism is analytic in $\mathrm{SB} \times \mathrm{SB}$ (see [Bos2] for more details). It follows that the set $A \cong$ is analytic. Hence, the set

$$
\tilde{A}=\left\{L \in[\mathbb{N}]: \exists Y \in A \text { with } U_{L} \cong Y\right\}=\Phi^{-1}(A \cong)
$$

is also analytic. The definition of the set $\tilde{A}$, the universality of the basis $\left(u_{n}\right)$ of the space $U$, and our starting assumptions on the set $A$ imply the following.

(P1) For every $L \in \tilde{A}$ there exists $Y \in A$ with $U_{L} \cong Y$.

(P2) For every $Y \in A$ there exists $L \in \tilde{A}$ with $Y \cong U_{L}$.

The space $[\mathbb{N}]$ is naturally identified as a closed subspace of the Baire space $\mathbb{N}^{\mathbb{N}}$. Hence, the set $\tilde{A}$ can be seen as an analytic subset of $\mathbb{N}^{\mathbb{N}}$. By [ $[\mathrm{Ke}$, Proposition 25.2], there exists a pruned tree $T$ on $\mathbb{N} \times \mathbb{N}$ such that $\tilde{A}=\operatorname{proj}[T]$. Notice that every non-empty node $t$ of $T$ is just a pair $(s, w)$ of finite sequences in $\mathbb{N}$ with $|s|=|t|$ and where $s$ is a strictly increasing finite sequence. Let $\mathrm{T}$ be the tree of non-empty 
sequences of $T$. For every $t=(s, w) \in \mathrm{T}$ we set $n_{t}=\max s$ and we define $x_{t}=u_{n_{t}}$. We also set $X=\overline{\operatorname{span}}\left\{x_{t}: t \in \mathrm{T}\right\}$. Using properties (P1) and (P2) above, it is easy to see that the tree $T$ and the family $\left(x_{t}\right)_{t \in \mathrm{T}}$ are as desired. The lemma is proved.

The second step towards the proof of Theorem 31 is based on a method of constructing Banach spaces introduced by R. C. James JJ and further developed by several authors (see, for instance, Bou1 and Bos2]). To describe it, we need to recall some terminology. Let $T$ be a tree on a set $\Lambda$ and consider the tree $\mathrm{T}$ of non-empty sequences of $T$. A subset $\mathfrak{s}$ of $\mathrm{T}$ is said to be a finite segment if there exist $s, t \in \mathrm{T}$ with $s \sqsubseteq t$ and such that $\mathfrak{s}=\{w \in \mathrm{T}: s \sqsubseteq w \sqsubseteq t\}$. If $\mathfrak{s}=\{w \in \mathrm{T}: s \sqsubseteq w \sqsubseteq t\}$ is a finite segment, then we set $\min \mathfrak{s}=s$. Two finite segments $\mathfrak{s}_{1}$ and $\mathfrak{s}_{2}$ of $\mathrm{T}$ are said to be incomparable if the nodes min $\mathfrak{s}_{1}$ and min $\mathfrak{s}_{2}$ are incomparable with respect to the partial order $\sqsubseteq$ of extension.

Definition 34 ( $\mathrm{AD}, \S 4.1)$. Let $\mathfrak{X}=\left(X, \Lambda, T,\left(x_{t}\right)_{t \in T}\right)$ be a Schauder tree basis. The $\ell_{2}$ Baire sum of $\mathfrak{X}$, denoted by $T_{2}^{\mathfrak{X}}$, is defined to be the completion of $c_{00}(T)$ equipped with the norm

$$
\|z\|_{T_{2}^{x}}=\sup \left\{\left(\sum_{i=0}^{l}\left\|\sum_{t \in \mathfrak{s}_{i}} z(t) x_{t}\right\|_{X}^{2}\right)^{1 / 2}\right\},
$$

where the above supremum is taken over all finite families $\left(\mathfrak{s}_{i}\right)_{i=0}^{l}$ of pairwise incomparable, finite segments of $T$.

Let $\mathfrak{X}=\left(X, \Lambda, T,\left(x_{t}\right)_{t \in \mathrm{T}}\right)$ be a Schauder tree basis and consider the correspond$\operatorname{ing} \ell_{2}$ Baire sum $T_{2}^{\mathfrak{X}}$. Let $\left(e_{t}\right)_{t \in \mathrm{T}}$ be the standard Hamel basis of $c_{00}(\mathrm{~T})$. We fix a bijection $h: \mathrm{T} \rightarrow \mathbb{N}$ such that for every pair $t, s \in \mathrm{T}$ we have that $h(t)<h(s)$ if $t \sqsubset s$. If $\left(e_{t_{n}}\right)$ is the enumeration of $\left(e_{t}\right)_{t \in \mathrm{T}}$ according to $h$, then it is easy to verify that the sequence $\left(e_{t_{n}}\right)$ defines a normalized bi-monotone Schauder basis of $T_{2}^{\mathfrak{X}}$. For every $\sigma \in[T]$, also let $\mathcal{X}_{\sigma}=\overline{\operatorname{span}}\left\{e_{\sigma \mid n}: n \geq 1\right\}$. It is also easily seen that the space $\mathcal{X}_{\sigma}$ is isometric to $X_{\sigma}$ and, moreover, it is 1-complemented in $T_{2}^{\mathfrak{X}}$ via the natural projection $P_{\sigma}: T_{2}^{\mathfrak{X}} \rightarrow \mathcal{X}_{\sigma}$.

Now let $Y$ be a subspace of $T_{2}^{\mathfrak{X}}$. Assume that there exist $\sigma \in[T]$ and a further subspace $Y^{\prime}$ of $Y$ such that the operator $P_{\sigma}: Y^{\prime} \rightarrow \mathcal{X}_{\sigma}$ is an isomorphic embedding. In such a case, the subspace $Y$ contains information about the Schauder tree basis $\mathfrak{X}=\left(X, \Lambda, T,\left(x_{t}\right)_{t \in \mathrm{T}}\right)$. On the other hand, there are subspaces of $T_{2}^{\mathfrak{X}}$ which are "orthogonal" to every $\mathcal{X}_{\sigma}$. We give them a special name, as follows.

Definition 35 ([AD], Definition 14). Let $\mathfrak{X}=\left(X, \Lambda, T,\left(x_{t}\right)_{t \in T}\right)$ be a Schauder tree basis and let $Y$ be a subspace of $T_{2}^{\mathfrak{X}}$. We say that $Y$ is $X$-singular if for every $\sigma \in[T]$ the operator $P_{\sigma}: Y \rightarrow \mathcal{X}_{\sigma}$ is strictly singular.

In $\mathrm{AD}$ the class of $X$-singular subspaces of $T_{2}^{\mathfrak{X}}$ was extensively analyzed. What we need, in order to finish the proof of Theorem 31, is the following structural result (see [AD, Theorem 24]).

Theorem 36. Let $\mathfrak{X}=\left(X, \Lambda, T,\left(x_{t}\right)_{t \in \mathrm{T}}\right)$ be a Schauder tree basis and let $Y$ be an $X$-singular subspace of $T_{2}^{\mathfrak{X}}$. Then $Y$ does not contain an isomorphic copy of $\ell_{1}$.

As a matter of fact, stronger properties than the one asserted by Theorem 36 are known (but any extra information is of no use in the argument below). We are ready to proceed to the proof of Theorem 31 . 
Proof of Theorem 31. Let $A$ be as in the statement of the theorem. By Lemma 33 . there exists a Schauder tree basis $\mathfrak{X}=\left(X, \Lambda, T,\left(x_{t}\right)_{t \in \mathrm{T}}\right)$ such that the following are satisfied.

(1) For every $Y \in A$ there exists $\sigma \in[T]$ with $Y \cong X_{\sigma}$.

(2) For every $\sigma \in[T]$ there exists $Y \in A$ with $X_{\sigma} \cong Y$. In particular, for every $\sigma \in[T]$ the space $X_{\sigma}$ is non-universal.

Consider the $\ell_{2}$ Baire sum $T_{2}^{\mathfrak{X}}$ of this Schauder tree basis $\mathfrak{X}$. We claim that the space $T_{2}^{\mathfrak{X}}$ is the desired one. Indeed, notice that $T_{2}^{\mathfrak{X}}$ has a Schauder basis and, by property (1) above, it contains every $Y \in A$ as a complemented subspace. What remains is to check that $T_{2}^{\mathfrak{X}}$ is non-universal.

We argue by contradiction. So, assume that there exists a subspace $E$ of $T_{2}^{\mathfrak{x}}$ which is isomorphic to $C\left(2^{\mathbb{N}}\right)$. Let $E^{\prime}$ be a subspace of $E$ which is isomorphic to $\ell_{1}$. By Theorem [36, we see that $E^{\prime}$ is not $X$-singular. It follows that there exist $\sigma \in[T]$ and an additional subspace $E^{\prime \prime}$ of $E^{\prime}$ such that $P_{\sigma}: E^{\prime \prime} \rightarrow \mathcal{X}_{\sigma}$ is an isomorphic embedding. Clearly we may additionally assume that $E^{\prime \prime}$ is isomorphic to $\ell_{1}$. Now consider the operator $P_{\sigma}: E \rightarrow \mathcal{X}_{\sigma}$. What we have just proved is that the operator $P_{\sigma}: E \rightarrow \mathcal{X}_{\sigma}$ fixes a copy of $\ell_{1}$. By Theorem 8 , we get that $\left.P_{\sigma}\right|_{E}$ must also fix a copy of $C\left(2^{\mathbb{N}}\right)$. This implies that the space $X_{\sigma}$ is universal, which is a contradiction by property (2) above. Having arrived at the desired contradiction, the proof of Theorem 31 is completed.

\section{The MAIN RESUlts}

This section is devoted to the proofs of Theorem 1, Theorem 3, Corollary 5 and Corollary [6] stated in the introduction. We start with the proof of Theorem 3 ,

Proof of Theorem 3. Let $A$ be an arbitrary analytic subset of NU. We apply Corollary 27 to the set $A$ and we get an analytic subset $A^{\prime}$ of NU such that

(1) every $Y \in A^{\prime}$ has a Schauder basis and

(2) for every $X \in A$ there exists $Y \in A^{\prime}$ containing an isometric copy of $X$.

By (1) above, we may apply Theorem 31 to the set $A^{\prime}$ and we get a non-universal space $Z$, with a Schauder basis, containing an isomorphic copy of every $Y \in A^{\prime}$. Invoking (2), we see that the space $Z$ is universal and for the class $A$. The proof is completed.

Proof of Theorem 1. As we have already indicated in the introduction, part (i) implies both (ii) and (iii). To see (ii) $\Rightarrow\left(\right.$ i), let $\mathcal{C}$ be a subset of SB and let $\xi<\omega_{1}$ be such that

$$
\sup \left\{\phi_{\mathrm{NU}}(X): X \in \mathcal{C}\right\} \leq \xi .
$$

By Theorem 12(i) and Lemma 10(i), the class $A_{\xi}=\left\{X \in \mathrm{NU}: \phi_{\mathrm{NU}}(X) \leq \xi\right\}$ is Borel and clearly $\mathcal{C} \subseteq A_{\xi}$. By Theorem [3, there exists a non-universal space $Y$ which is universal for the class $A_{\xi}$. A fortiori, the space $Y$ is universal for the class $\mathcal{C}$. That is, part (i) is satisfied. For the implication (iii) $\Rightarrow(\mathrm{i})$ we argue similarly.

Proof of Corollary 5. Let $\mathcal{C}$ be an isomorphic invariant class of separable Banach spaces. As we have already mentioned, if the class $\mathcal{C}$ is Bossard generic, then $\mathcal{C}$ is also Bourgain generic. To see the converse, assume that $\mathcal{C}$ is not Bossard generic. Hence, we may find an analytic subset $A$ of NU such that for every $X \in \mathcal{C}$ there exists $Z \in A$ with $X \cong Z$. We apply Theorem 3 and we get a non-universal separable Banach space $Y$ containing an isomorphic copy of every $Z \in A$. Clearly, 
the space $Y$ witnesses the fact that the class $\mathcal{C}$ is not Bourgain generic. Therefore, the two notions coincide, as desired.

Proof of Corollary 6. Fix $\lambda>1$. The family $\left\{Y_{\xi}^{\lambda}: \xi<\omega_{1}\right\}$ will be constructed by transfinite recursion on countable ordinals. As the first step is identical to the general one, we may assume that for some countable ordinal $\xi$ and every $\zeta<\xi$ the space $Y_{\zeta}^{\lambda}$ has been constructed. We set

$$
C=\left\{X \in \mathrm{NU}: \phi_{\mathrm{NU}}(X) \leq \xi\right\} \cup\left\{Y_{\zeta}^{\lambda}: \zeta<\xi\right\} .
$$

By Theorem 12(i) and Lemma 10(i), the set $C$ is an analytic subset of NU. We apply Theorem 3 and we get a separable non-universal space $X$ which is universal for the class $C$. We define $Y_{\xi}^{\lambda}$ to be the space $\mathcal{L}_{\lambda}[X]$. This completes the recursive construction. Using Theorem [16] and Proposition [13, it is easily verified that the family $\left\{Y_{\xi}^{\lambda}: \xi<\omega_{1}\right\}$ is as desired.

\section{Further Strongly Bounded Classes}

This section is devoted to the proof of Theorem 7 stated in the introduction. To this end, we need the following analogue of Theorem 31 for the class $\mathrm{NC}_{X}$.

Theorem 37 ([AD], Theorem 87). Let $X$ be a minimal Banach space. Also let $A$ be an analytic subset of $\mathrm{NC}_{X}$ such that every $Y \in A$ has a Schauder basis. Then there exists a space $V \in \mathrm{NC}_{X}$, with a Schauder basis, that contains every $Y \in A$ as a complemented subspace.

We are ready to proceed to the proof of Theorem 7.

Proof of Theorem 7 . Let $X$ be a minimal Banach space not containing an isomorphic copy of $\ell_{1}$ and let $A$ be an arbitrary analytic subset of $\mathrm{NC}_{X}$. We apply Corollary 29 to the set $A$ and we get an analytic subset $A^{\prime}$ of $\mathrm{NC}_{X}$ such that

(1) every $Y \in A^{\prime}$ has a Schauder basis and

(2) for every $Z \in A$ there exists $Y \in A^{\prime}$ containing an isometric copy of $Z$.

Applying Theorem 37 to the set $A^{\prime}$ and invoking (1) above, we see that there exists a space $V \in \mathrm{NC}_{X}$, with a Schauder basis, which is universal for the class $A^{\prime}$. By (2) above, we get that the space $V$ is universal for the class $A$ as well. The proof is completed.

We close this section by giving the following analogue of Corollary 6. Its proof is identical to that of Corollary 6.

Corollary 38. Let $X$ be a minimal Banach space with a Schauder basis and not containing $\ell_{1}$. Then, for every $\lambda>1$, there exists a family $\left\{Y_{\xi}^{\lambda}: \xi<\omega_{1}\right\}$ of separable Banach spaces with the following properties.

(i) For every $\xi<\omega_{1}$ the space $Y_{\xi}^{\lambda}$ is $\mathcal{L}_{\infty, \lambda+}$ and does not contain a copy of $X$.

(ii) If $\xi<\zeta<\omega_{1}$, then $Y_{\xi}^{\lambda}$ is contained in $Y_{\zeta}^{\lambda}$.

(iii) If $Z$ is a separable space with $\phi_{\mathrm{NC}_{X}}(Z) \leq \xi$, then $Z$ is contained in $Y_{\xi}^{\lambda}$. 


\section{REFERENCES}

[AD] S. A. Argyros and P. Dodos, Genericity and amalgamation of classes of Banach spaces, Adv. Math., 209 (2007), 666-748. MR2296312 (2008f:46014)

[Bos1] B. Bossard, Théorie descriptive des ensembles en géométrie des espaces de Banach, Thèse, Univ. Paris VI, 1994.

[Bos2] B. Bossard, A coding of separable Banach spaces. Analytic and co-analytic families of Banach spaces, Fund. Math., 172 (2002), 117-152. MR1899225 (2003d:46016)

[Bou1] J. Bourgain, On separable Banach spaces, universal for all separable reflexive spaces, Proc. AMS, 79 (1980), 241-246. MR565347 (81f:46021)

[Bou2] J. Bourgain, New classes of $\mathcal{L}_{p}$ Spaces, Lecture Notes in Math., 889, Springer-Verlag, 1981. MR639014 (83j:46028)

[BD] J. Bourgain and F. Delbaen, A class of special $\mathcal{L}_{\infty}$-spaces, Acta Math., 145 (1980), 155176. MR 590288 (82h:46023)

[BP] J. Bourgain and G. Pisier, A construction of $\mathcal{L}_{\infty}$-spaces and related Banach spaces, Bol. Soc. Brasil. Mat., 14 (1983), 109-123. MR.756904 (86b:46021)

[DF] P. Dodos and V. Ferenczi, Some strongly bounded classes of Banach spaces, Fund. Math., 193 (2007), 171-179. MR2282714 (2008f:46016)

[Ga] I. Gasparis, Operators on C $[0,1]$ preserving copies of asymptotic $\ell_{1}$ spaces, Math. Annalen, 333 (2005), 831-858. MR2195147 (2007a:46006)

[J] R. C. James, A separable somewhat reflexive Banach space with non-separable dual, Bull. AMS, 80 (1974), 738-743. MR0417763 (54:5811)

[JRZ] W. B. Johnson, H. P. Rosenthal and M. Zippin, On bases, finite-dimensional decompositions and weaker structures in Banach spaces, Israel J. Math., 9 (1971), 488-506. MR 0280983 (43:6702)

[Ke] A. S. Kechris, Classical Descriptive Set Theory, Grad. Texts in Math., 156, SpringerVerlag, 1995. MR 1321597 (96e:03057)

[Ki] S. V. Kisliakov, On spaces with "small" annihilators, Zap. Naucn. Sem. Leningrad. Otdel. Math. Institute Steklov (LOMI), 65 (1976), 192-195. MR0500098(58:17804)

[LP1] J. Lindenstrauss and A. Pełczyński, Absolutely summing operators in $\mathcal{L}_{p}$ spaces and their applications, Studia Math., 29 (1968), 275-326. MR0231188 (37:6743)

[LP2] J. Lindenstrauss and A. Pełczyński, Contributions to the theory of classical Banach spaces, J. Funct. Anal., 8 (1971), 225-249. MR0291772 (45:863)

[LT] J. Lindenstrauss and L. Tzafriri, Classical Banach spaces vol. I: Sequence spaces, Ergebnisse, vol. 92, Springer, 1977. MR 0500056 (58:17766)

[Mi] A. A. Milutin, Isomorphisms of spaces of continuous functions on compacts of power continuum, Tieoria Func. (Kharkov), 2 (1966), 150-156.

[OS] E. Odell and Th. Schlumprecht, A separable reflexive space universal for the uniformly convex Banach spaces, Math. Annalen, 335 (2006), 901-916. MR2232021 (2007f:46012)

[OSZ] E. Odell, Th. Schlumprecht and A. Zsák, Banach spaces of bounded Szlenk index, Studia Math., 183 (2007), 63-97. MR2360257

[P] A. Pełczyński, Universal bases, Studia Math., 32 (1969), 247-268. MR0241954 (39:3290)

[Pi] G. Pisier, Counterexamples to a conjecture of Grothendieck, Acta Math., 151 (1983), 181208. MR723009 (85m:46017)

[Ro1] H. P. Rosenthal, On factors of $C([0,1])$ with non-separable dual, Israel J. Math., 13 (1972), 361-378. MR0388063 (52:8900)

[Ro2] H. P. Rosenthal, A characterization of Banach spaces containing $\ell_{1}$, Proc. Nat. Acad. Sci. USA, 71 (1974), 2411-2413. MR0358307 (50:10773)

[Ro3] H. P. Rosenthal, The Banach spaces $C(K)$, Handbook of the Geometry of Banach Spaces, Volume 2, Edited by W. B. Johnson and J. Lindenstrauss, Elsevier, 2003. MR:1999613 (2004e:46001)

[Ts] B. S. Tsirelson, Not every Banach space contains $\ell_{p}$ or $c_{0}$, Functional Anal. Appl., 8 (1974), 138-141. MR0350378(50:2871)

Department of Mathematics, Faculty of Applied Sciences, National Technical University of Athens, Zografou Campus, 157 80, Athens, Greece

E-mail address: pdodos@math.ntua.gr 